\section{SANDIA REPORT}

SAND95-1950 - UC-910

Unlimited Release

Printed August 1995
RECEIVED

OCT 111995

OSTI

\title{
Ultra-High-Speed Optical and Electronic Distributed Devices
}

Vincent M. Hietala, Thomas A. Plut, Stanley H. Kravitz, G. Allen Vawter, Joel R. Wendt, Marcelino G. Armendariz

Prepared by

Sandia National Laboratories

Albuquerque, New Mexico 87185 and Livermore, California 94550

for the United States Department of Energy

under Contract DE-ACO4-94AL85000

Approved for public release; cistribution is unlimited.

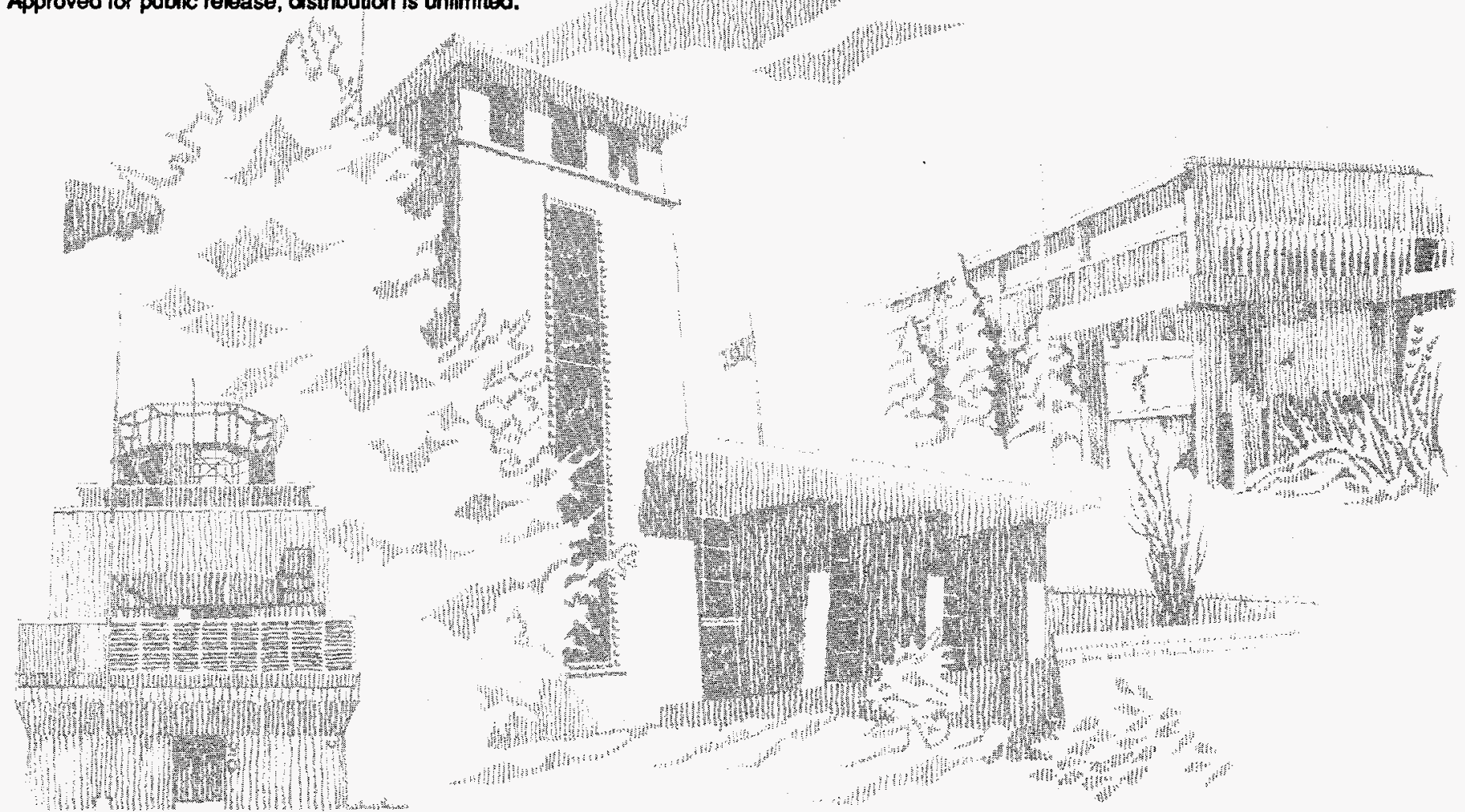


Issued by Sandia National Laboratories, operated for the United States Department of Energy by Sandia Corporation.

NOTICE: This report was prepared as an account of work sponsored by an agency of the United States Government. Neither the United States Government nor any agency thereof, nor any of their employees, nor any of their contractors, subcontractors, or their employees, makes any warranty, express or implied, or assumes any legal liability or responsibility for the accuracy, completeness, or usefulness of any information, apparatus, product, or process disclosed, or represents that its use would not infringe privately owned rights. Reference herein to any specific commercial product, process, or service by trade name, trademark, manufacturer, or otherwise, does not necessarily constitute or imply its endorsement, recommendation, or favoring by the United States Government, any agency thereof or any of their contractors or subcontractors. The views and opinions expressed herein do not necessarily state or reflect those of the United States Government, any agency thereof or any of their contractors.

Printed in the United States of America. This report has been reproduced directly from the best available copy.

Available to DOE and DOE contractors from Office of Scientific and Technical Information

PO Box 62

Oak Ridge, TN 37831

Prices available from (615) 576-8401, FTS 626-8401

Available to the public from

National Technical Information Service

US Department of Commerce

5285 Port Royal Rd

Springfield, VA 22161

NTIS price codes

Printed copy: A03

Microfiche copy: A01 


\section{DISCLAIMER}

Portions of this document may be illegible in electronic image products. Images are produced from the best available original document. 


\title{
ULTRA-HIGH-SPEED OPTICAL AND ELECTRONIC DISTRIBUTED DEVICES
}

\author{
Vincent M. Hietala, Thomas A. Plut, Stanley H Kravitz, and G. Allen Vawter, \\ and Joel R. Wendt \\ Compound Semiconductor Technology Department
}

Marcelino G. Armendariz

Advanced Devices \& Applications Department

Sandia National Laboratories

Albuquerque, New Mexico 87185-0603

\begin{abstract}
This report summarizes work on the development of ultra-high-speed semiconductor optical and electronic devices. High-speed operation is achieved by velocity matching the input stimulus to the output signal along the device's length. Electronic devices such as field-effect transistors (FET's), should experience significant speed increases by velocity matching the electrical input and output signals along the device. Likewise, optical devices, which are typically large, can obtain significant bandwidths by velocity matching the light being generated, detected or modulated with the electrical signal on the device's electrodes. The devices discussed in this report utilize truly distributed electrical design based on slow-wave propagation to achieve velocity matching.
\end{abstract}

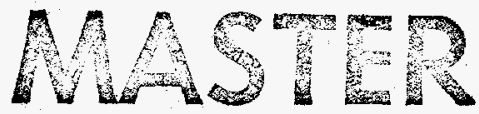




\section{Acknowledgment}

The authors are indebted for the technical processing assistance of Albert G. Baca, and Arnold J. Howard. The authors also wish to thank the expert device fabrication by Beth $A$. Fuchs and Burt Snipes, and device characterization by Thomas M. Bauer, Charles T. Fuller, Charlie E. Sandoval, and Mark S. Housel. 


\section{Contents}

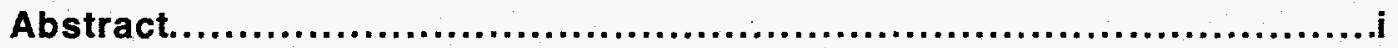

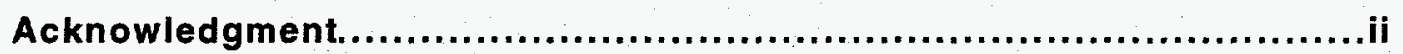

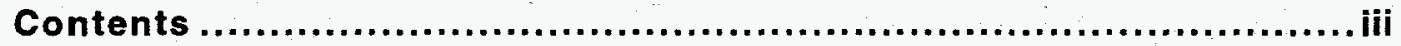

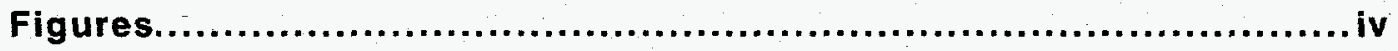

Tables........................................................................ iv

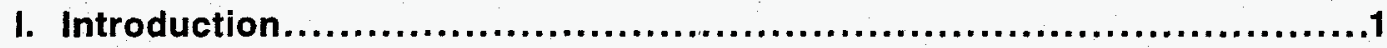

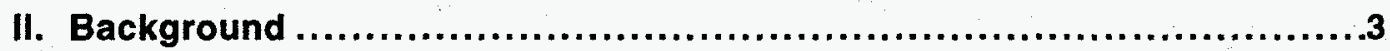

Velocity Matching................................................................................ 3

Slow-wave Transmission Lines............................................................ 5

III. Distributed Optical Phase Modulators...................................8

IV. Distributed Field-Effect Transistors.................................9

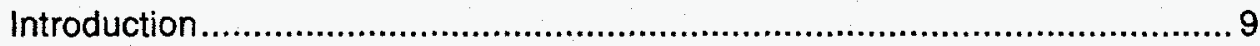

Theory of Operation............................................................................. 9

DFET Fabrication ............................................................................ 14

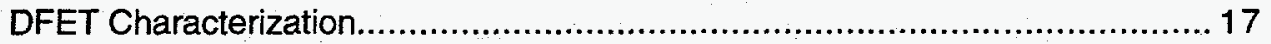

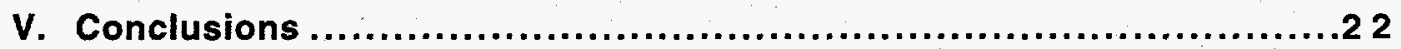

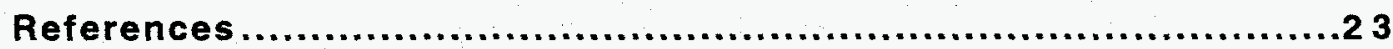

Appendix A. Reprint of "High-performance GaAs/AlGaAs optical phase modulators for microwave/photonic integrated circuits" ..........25

ABSTRACT 2 MOTIVATION

Appendix B. Reprint of invited presentation at MTT-S "High-Performance GaAs/AIGaAs Optical Phase Modulators for Microwave Photonic Integrated Circuits"...................................................... 5

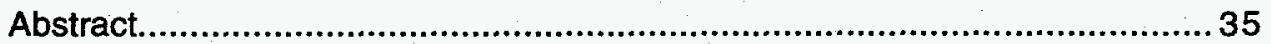

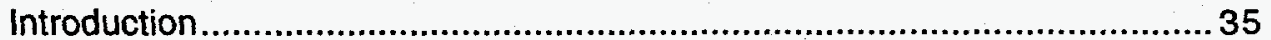

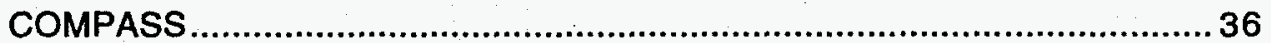

Depletion-Edge-Translation Modulator........................................................37

Distributed Electrode Design ................................................................... 38

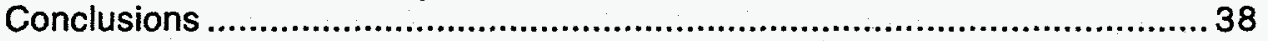

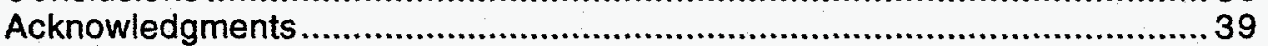

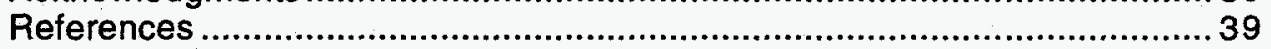

Appendix C. LDRD Summary............................................4 3 


\section{Figures}

Fig. 1. Surface plot of normalized response of a distributed device as a function normalized iength and RF index difference.

Fig. 2. Quasi-TEM equivalent circuit of a slow-wave transmission line. ................................... 6

Fig. 3. Quasi-static field plots to determine electrical propagation.......................................... 7

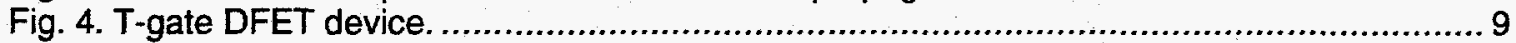

Fig. 5. Schematic of coupled three line DFET Model....................................................... 10

Fig. 6. Field plots from electromagnetic simulations of the DFET ......................................13

Fig. 7. DFET process sequence. Dimensions of final device are in micrometers ......................15

Fig. 8. SEM micrographs of the DFET........................................................................ 16

Fig. 9. DC electrical characteristics of a lumped $8 \times 120$ DFET $(100 \mu \mathrm{m}$ gate width) $\ldots \ldots \ldots \ldots \ldots \ldots . . \ldots 17$

Fig. 10. Measured and modeled S-parameters of a lumped $8 \times 120$ DFET (100 $\mu \mathrm{m}$ gate width).. 18

Fig. 11. Measured response of a $100 \mu \mathrm{m}$ gate width $8 \times 120$ DFET. Dash-dot line has a slope of -20 $\mathrm{dB} / \mathrm{dec}$.

Fig. 12. Equivalent circuit of lumped DFET .......................................................................... 19

Fig. 13. Comparison between measured (points) and theoretical (line) magnitude of $S_{21} \ldots \ldots \ldots .21$

Fig. 14. Comparison between measured (points) and theoretical (line) magnitude of the unilateral transducer gain, IUI

\section{Tables}

Table 1. Half wavelengths at various frequencies in GaAs. 1

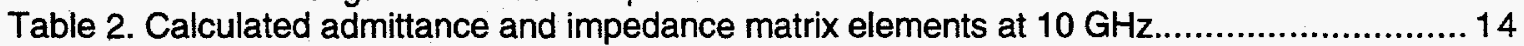
Table 3. DFET material growth specification. UID and SI mean unintentionally doped and semiinsulating, respectively.

Table 4. Lumped DFET parameters fit $(0.05$ to $15 \mathrm{GHz})$ to the equivalent circuit shown in Fig. 12.20 


\section{Introduction}

The demand for faster electronic and optoelectronic devices does not appear to be abating. This demand is fueled in part, by the desire for faster data exchange whether digital or analog for which a significant practical limit has not yet been achieved. Presently, device speeds extending into the millimeter wave regime $(30 \mathrm{GHz}<f<300 \mathrm{GHz})$ are not uncommon, though they are difficult to produce with present technologies. Additionally, in the foreseeable future, a real need for devices with operating speeds in excess of $100 \mathrm{GHz}$ is expected. It is the purpose of this work to explore a possible solution for meeting this future need.

To date, most high speed devices have been produced by incremental engineering (i.e. size scaling) of low frequency designs. This approach will be ineffective as the operating wavelength becomes small compared to the minimum device size. This limit can be understood by comparing the wavelength in a semiconductor to typical device sizes. The electromagnetic wavelength within a semiconductor media is

$$
\lambda=\frac{c}{f \sqrt{\varepsilon_{r}}}
$$

where $c$ is the speed of light, $f$ is the operating frequency, and $\varepsilon_{r}$ is the semiconductor's dielectric constant. Using (1), Table 1 shows the length of a half wavelength in GaAs $\left(\varepsilon_{r}=13\right)$ at various frequencies. The half-wavelength length scale is important as it represents a complete $\pi$ phase shift often resulting in total destructive interference within the device.

\begin{tabular}{c|c}
\hline Frequency, $f(\mathrm{GHz})$ & Half Wavelength, $\lambda / 2(\mu \mathrm{m})$ \\
\hline $10 \mathrm{GHz}$ & $4200 \mu \mathrm{m}$ \\
$100 \mathrm{GHz}$ & $420 \mu \mathrm{m}$ \\
$1 \mathrm{THz}$ & $42 \mu \mathrm{m}$ \\
\hline
\end{tabular}

Table 1. Half wavelengths at various frequencies in GaAs.

In practice, device designs need to be much more conservative with $\lambda / 8$ being a common limit. Either way, the size of power FETs and optoelectronic devices are commonly thousands of micrometers in order to achieve the required power or efficiency levels resulting in devices many wavelengths in size. Consequently, understanding distributed effects and velocity matching is critical in the design of high-performance high-speed devices.

This report summarizes the results of a Laboratory Directed Research and Development (LDRD) project performed at Sandia National Laboratories (case \#3512.040). The project summarized in this report was made up of two 
main efforts: high-speed high-efficiency optical phase modulators and distributed FETs. The first section of this report will demonstrate the general effect of velocity mismatch and discuss the basic operation of slow-wave transmission lines that are used as the basis to obtain the necessary velocity match in this work. Then brief sections on the project's phase modulator effort and distributed FET efforts will be presented. 


\section{Background}

\section{Velocity Matching}

To appreciate the need for velocity matching, it is informative to look at a simple example in some detail. Assume two traveling waves $A$ and $B$ exist within a hypothetical device with intrinsic propagation constants $\gamma_{A}$ and $\gamma_{B}$ respectively. Furthermore, assume the input to the device is represented by wave $A$, of the form

$$
A(z)=A_{o} e^{-\gamma_{A} z}
$$

and the output from the device is wave $B$. Only forward propagating waves as described by (2) will be assumed to exist within the device. These waves may represent voltages, currents, or some field quantity.

For a lumped device, the output would simply be the product of a coupling factor per unit length $\xi$, length $l$, and input signal:

$$
B_{l}(l)=\frac{\xi}{2} l A
$$

where the factor of $1 / 2$ is necessary to be consistent with the forward propagating wave assumption. The physical meaning of $\xi$ is device dependent. For distributed FETs, $\xi$ is the transconductance per unit length. For an optical phase modulator, $\xi$ is the figure of merit (FOM).

For a distributed device, the signal at the device's output is found by integrating the contribution to the output wave generated at each point multiplied by the appropriate propagation factor over the device's length:

$$
B(l)=\int_{0}^{l} \frac{\xi}{2} A(z) e^{-\gamma_{B}(l-z)} d z=\frac{\xi e^{-\gamma_{B} l}}{2\left(\gamma_{B}-\gamma_{A}\right)}\left[e^{\left(\gamma_{B}-\gamma_{A}\right) l}-1\right] .
$$

For purposes of this discussion, we now assume propagation along the device to be lossless making $\gamma_{B}=\mathrm{j} n_{B} \beta_{o}$ and $\gamma_{A}=\mathrm{j} n_{A} \beta_{o}$ where $\beta_{o}$ is the free space propagation constant $(\omega / c)$ and $n$ are the RF indexes. This assumption applied to (4) along with normalization to the lumped result (3), gives a device response, $R_{n}$, of

$$
R_{n}=\left|\frac{B(l)}{B_{l}(l)}\right|=\left|\frac{e^{j \Delta n \beta_{o} l}-1}{j \Delta n \beta_{o} l}\right|
$$

where $\Delta n$ is the difference between the two wave propagation indices $\left(n_{B}-n_{A}\right)$. 
A surface plot of Eq. (5) is shown in Fig. 1. In this plot the normalized response is shown as a function of normalized length (length $\left./ \lambda_{0}\right)$ and index difference $(\Delta n)$. This plot clearly shows the penalty of velocity mismatch in distributed device operation. When the length of the device is a fraction of a wavelength as in conventional low-frequency electronic components, a velocity match is not important as seen on the left side of the plot. Conversely, if the device is several wavelengths in length, velocity matching $(\Delta n=0)$ is critical as seen on the right side of the plot. Since velocity mismatch of FETs is typically much higher than the $|\Delta n|=2$ limit of this plot, it is easy to appreciate the $\lambda / 8$ guideline commonly used for FET design as discussed in the introduction. Clearly a velocity match is critical for large high-frequency devices.

It is important to realize that we have assumed no frequency dependence with the lumped device's response (3). This results, as shown in Fig. 1, with the distributed device's response always being less than that of the lumped device. In reality, the lumped device will suffer a roll off in response that is more rapid than that exhibited by a transmission line. This results in a normalized response that would appear in Fig. 1 as a proportional increase towards the right side of the plot. This may appear as a contradiction since the distributed device is made from the lumped device; however, parasitics of the lumped device are "absorbed" within the distributed device allowing for a significant increase in bandwidth.

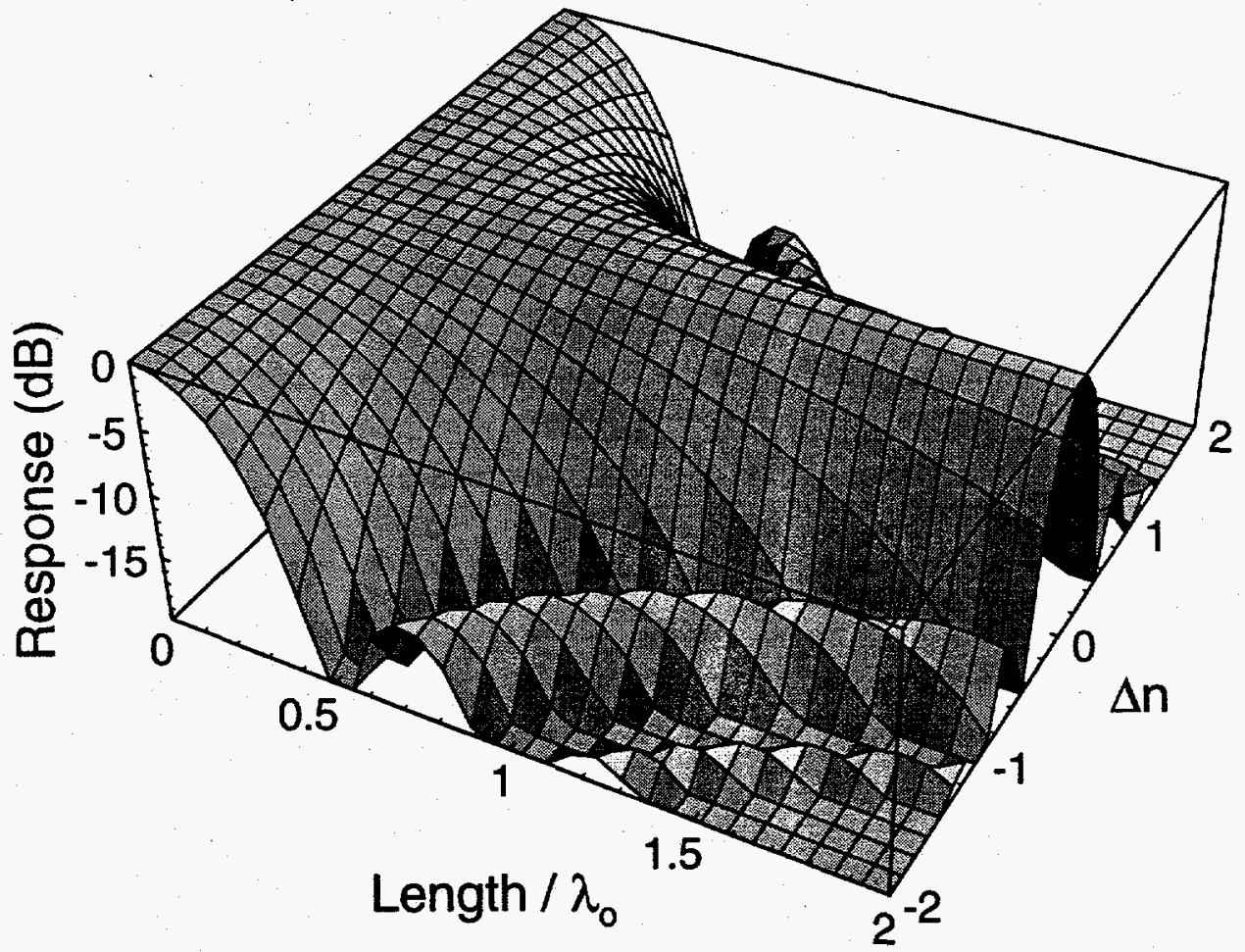

Fig. 1. Surface plot of normalized response of a distributed device as a function normalized length and RF index difference. 


\section{Slow-wave Transmission Lines}

To achieve the necessary velocity match discussed in the previous section, slow-wave transmission lines are used. Slow wave propagation is readily achieved in transmission lines laid over semiconductor devices composed of heavily-doped layers. In fact, any transmission line laid in close proximity to a somewhat conductive layer will exhibit slow-wave propagation effects. As an example of this type of transmission line, slow-wave coplanar waveguides have been extensively studied.[1],[2] Such transmission lines require "full-wave" analysis for rigorous modeling.[3] Fortunately for our purposes, quasi-TEM analysis [4] satisfactorily describes these types of lines.

The line's impedance per unit length, $Z$, and admittance per unit length, $Y$, as shown in the equivalent circuit in Fig. 2, were determined by using a quasistatic finite element simulator from Ansoft.[5] A representative set of contour plots from this simulator is shown in Fig. 3 (see Appendix B for device details). The shunt admittance is found from a 2-D quasi-static electric field calculation in which the semiconductor layers are assigned a complex permittivity to account for conduction. The device's electrodes are assumed perfect conductors which provides simple boundary conditions for the solution of Laplace's equation. The resulting contour plot of the electrostatic potential is shown in Figure 3a. The capacitance per unit length, $C$, and the shunt admittance per unit length, $G$, can then be readily determined by integration over the field solution. The series impedance is similarly found by solving the quasi-magnetostatic problem where a longitudinal current is allowed to flow in all conductive layers. This results in a series inductance per unit length, $L$, and a resistance per unit length, $R$, which accounts for metal loss and power loss caused by the induced current in the semiconductor layers.

The plots shown in Fig. 3 illustrate the basic nature of slow-wave transmission lines. The highly conductive semiconductor layers beneath the electrodes effectively shields electric field penetration into the device's substrate. Therefore, the electrical energy storage $(\approx C)$ along the line is similar to a conventional shield coplanar waveguide. On-the-other-hand, the magnetic field can readily penetrate through the conductive semiconductor layers. This occurs since the skin depth in the semiconductor is typically much thicker than the epi-grown semiconductor layers even at the highest possible doping levels and at hundreds of $\mathrm{GHz}$ operating frequencies. This penetration of the magnetic field within the device's substrate greatly increase the magnetic energy storage along the transmission line. This increased energy storage along the line necessarily means a slow propagation velocity along the line. This situation is made apparent by considering the energy density and propagation speed contributions to power flow. High energy densities must correspond to relatively slower propagation at a fixed power flow. 


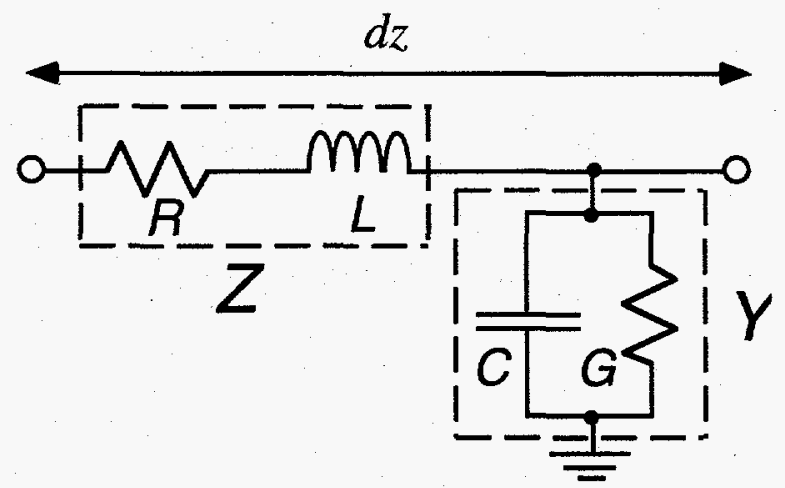

Fig. 2. Quasi-TEM equivalent circuit of a slow-wave transmission line.

With these equivalent circuit elements known, the transmission line's impedance $Z$ and admittance $Y$ per unit length can be determined by

and

$$
Z=R+j \omega L
$$

$$
Y=G+j \omega C .
$$

The complex propagation constant, $\gamma$, and the complex characteristic impedance, $Z_{0}$, can then be found:

$$
\gamma=\sqrt{Z Y}
$$

and

$$
Z_{o}=\sqrt{\frac{Z}{Y}} .
$$

The velocity of propagation of slow-wave transmission lines can be adjusted by changing design dimensions. For instance, simple changes in the insulating layer of an appropriately designed transmission line can result in a variation of the velocity of propagation over a few orders of magnitude. The characteristic impedance of these lines is also readily adjustable independent of the propagation characteristics. This is due to designers having the ability to independently select $L$ and $C$. 


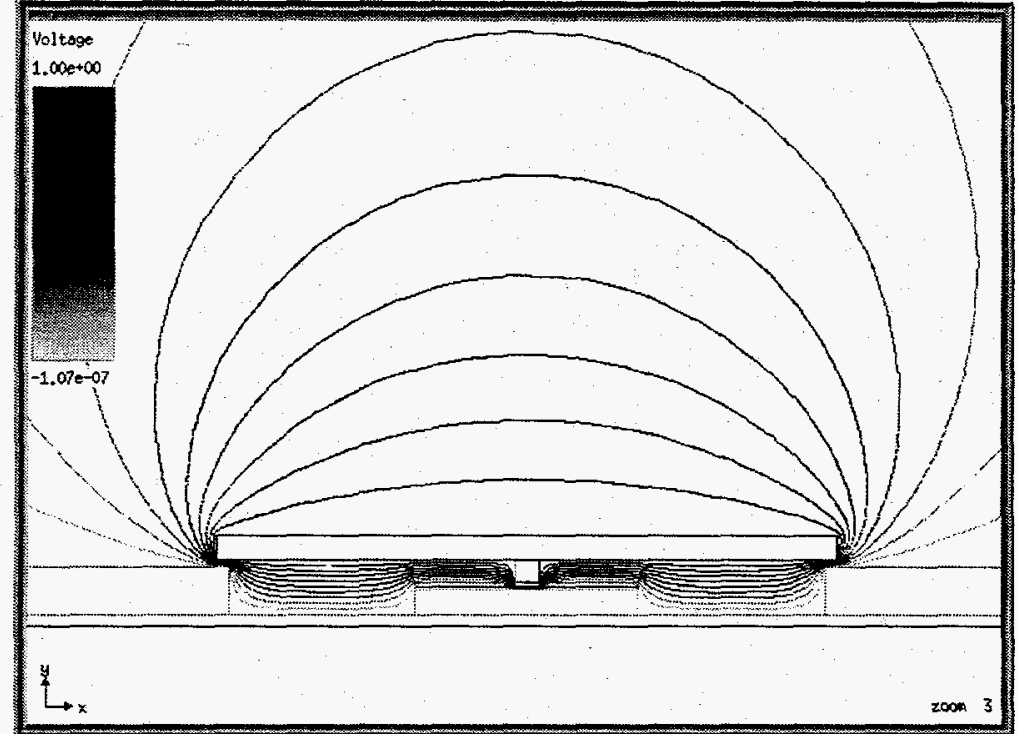

(a) Contours of constant electrostatic potential. From the admittance calculation of a transmission line.

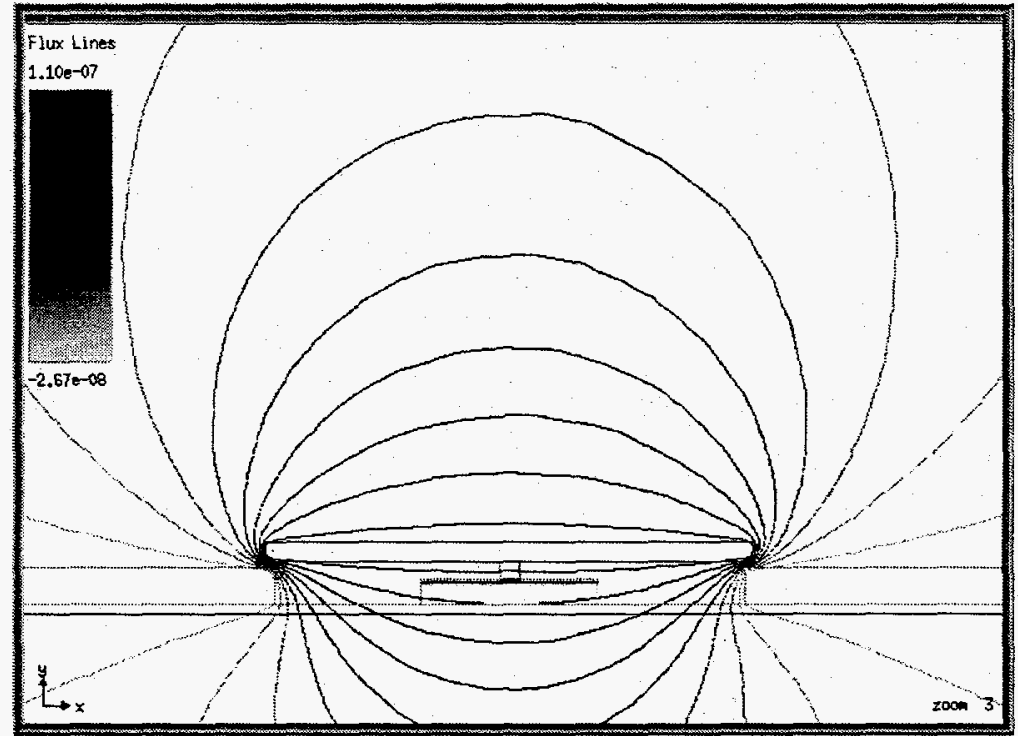

(b) Contours of constant longitudinal magnetic vector potential. From the impedance calculation of a transmission line.

Fig. 3. Quasi-static field plots to determine electrical propagation. 


\section{Distributed Optical Phase Modulators}

Optical phase modulators are a critical component in the fabrication of optoelectronic circuits. The paper reprint in Appendix B outlines a hypothetical optoelectronic circuit with emphasis on modulator performance. This paper demonstrates the need for high modulation efficiency and wide electrical bandwidth modulators. The high modulation efficiency required can be satisfied using a Depletion Edge Translation (DET) optical phase modulators. These modulators are based on the appropriate combination of both electro-optic and carrier effects to provide a maximum phase shift.[6]. Additionally, the modulated light is confined close to the junction's center providing a maximum field overlap and heavy doping levels provide a high electric field within the junction. These design techniques result in very high modulation efficiency, but they also cause a large terminal capacitance. If conventional lumped designs are used, this capacitance greatly limits device speed. By using the distributed techniques discussed in the previous section, we have been able to overcome this limitation and produce modulators simultaneously exhibiting very high modulation efficiencies and large electrical bandwidths.

Distributed optical phase modulators have been demonstrated with a very high, $60 \% / \mathrm{N} \cdot \mathrm{mm}$, modulation efficiency and a $10 \mathrm{GHz}$ bandwidth. Additionally, these modulators are easily integrated, since passive waveguides and phase modulators can be readily fabricated on the same substrate. Details on these modulators are given in Appendix A and B. 


\section{Distributed Field-Effect Transistors}

\section{Introduction}

The benefits of distributed electronic device operation has been understood and utilized for decades.[7] Though many traveling-wave solid-state devices based in exact analogy to the traveling-wave tube have been proposed[8] and even demonstrated[9], the traveling-wave or Distributed Field-Effect Transistor (DFET) have never been successfully implemented in a practical form. The DFET was first proposed by G. W. Mclver in 1965.[10] Almost 20 years later, the first detailed theory of the DFET was developed by Podgorski and Wei in 1982.[11] The first notable realization of the DFET was in 1985 by Holden et al[12] which demonstrated feasibility, but lacked practical performance.

A rigorous full-wave treatment of the DFET appeared in 1987 by Heinrich and Hartnagel.[13] At this point it became evident that propagation losses along the typically very small gate of a FET were prohibitively large and minimization of this propagation loss is the goal of distributed operation. As a result, a distributed FET with reduced gate loss by low-loss power distribution was realized [14] and the fabrication of large T-gate structures was attempted [15]. The objective of this project is the development of a suitable low-loss FET electrode structure amenable to true traveling-wave operation.

The structure we pursued is shown schematically in Fig. 4. Gate area is maximized in-order-to minimize propagation loss. FETs with T-gate widths $\mathrm{S}=4.5 \mu \mathrm{m}$ and $10.5 \mu \mathrm{m}$, both with $1 \mu \mathrm{m}$ electrical gate length were fabricated. Additionally, drain and source widths $W=10.5,35.5$ and $120.5 \mu \mathrm{m}$ were explored for a total of 6 different device types. The naming convention used throughout this report is DFET SxW. All data presented in this report is from the device set with $8 \mu \mathrm{m}$ gate-T width and $120 \mu \mathrm{m}$ source/drain width. (DFET $8 \times 120)$.

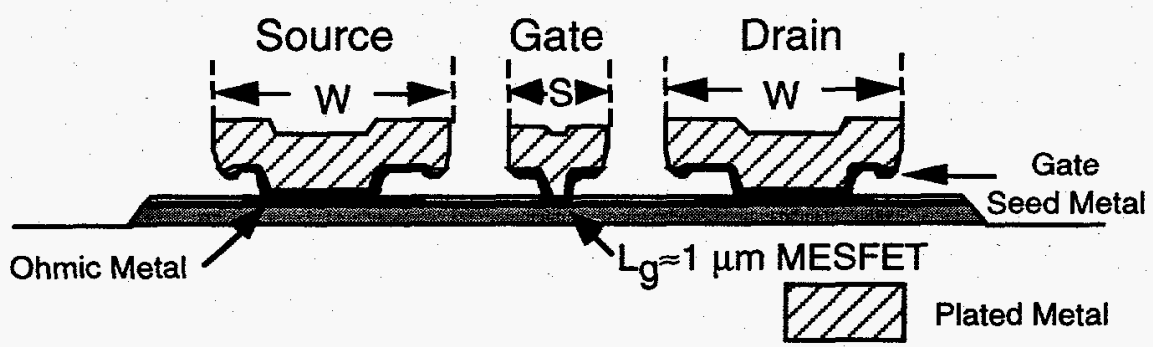

Fig. 4. T-gate DFET device.

\section{Theory of Operation}

The analysis presented here follows the coupled active line theory presented in [16], but it is extended to properly include magnetic coupling terms. 


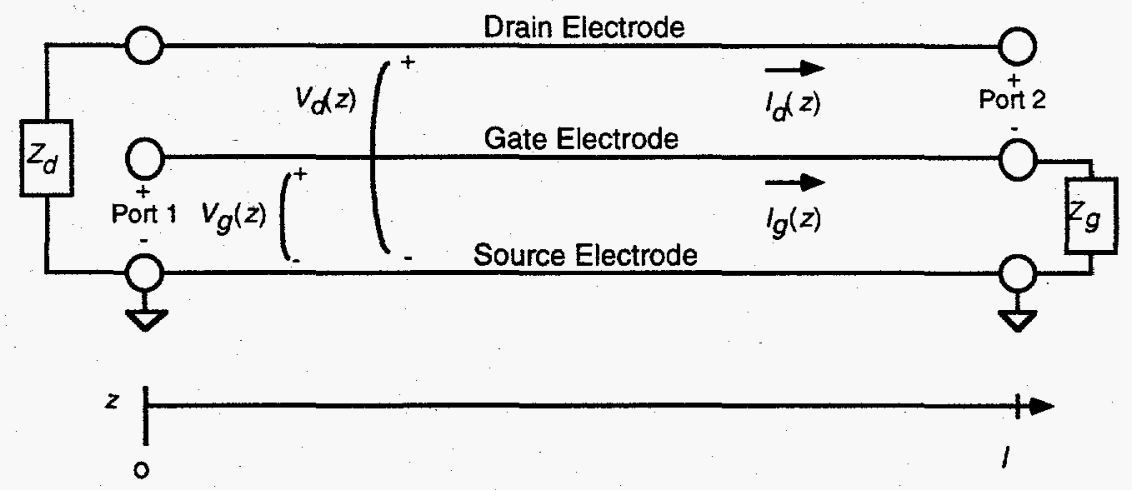

Fig. 5. Schematic of coupled three line DFET Model.

The Ansoft's Maxwell 2-D simulator allows for the calculation of equivalent parameters for multiconductor transmission lines. The simulation provides four matrix lumped element results at a given frequency - $\mathbf{L}, \mathbf{R}, \mathbf{C}$, and $\mathbf{G}$, representing the self- and mutual-inductances; losses due to series resistance; and the capacitance and conductance between lines; respectively. These parameters enter in to the multiconductor telegrapher's equations as:

and

$$
\frac{\partial \vec{V}}{d z}=-\mathbf{Z} \vec{I}
$$

in which:

$$
\frac{\partial \vec{I}}{d z}=-\mathbf{Y} \vec{V}
$$

and

$$
\mathbf{Z}=\mathbf{R}+j \omega \mathbf{L}
$$

$$
\mathbf{Y}=\mathbf{G}+j \omega \mathbf{C} \text {. }
$$

The $\mathbf{Z}$ and $\mathbf{Y}$ matrix are diagonally symmetric for passive multiconductor transmission lines, indicating that the mutual effects between any two signal lines are identical. However, when the coupling between the lines is made active, this symmetry does not exist. In the case of a DFET, to first order, the active element in the equivalent circuit model is a current source from the drain to source electrode controlled by the electric potential from the gate to source $\left(I_{d s}=g_{m}, V_{g s}\right)$. Assuming the voltage and current vectors along the FET are defined as:

$$
\vec{V}=\left[\begin{array}{l}
V_{d} \\
V_{g}
\end{array}\right] \text { and } \vec{I}=\left[\begin{array}{l}
I_{d} \\
I_{g}
\end{array}\right],
$$

the $\mathbf{Z}$ and $\mathbf{Y}$ matrices for the DFET become: 


$$
\mathbf{Z}=\left[\begin{array}{ll}
z_{d} & z_{m} \\
z_{m} & z_{g}
\end{array}\right]
$$

and

$$
\mathbf{Y}=\left[\begin{array}{cc}
y_{d} & y_{m}+g_{m} \\
y_{m} & y_{g}
\end{array}\right]
$$

where $g_{m}$ is the transconductance.

Combining (11) and (12) gives:

$$
\frac{\partial^{2} \vec{V}}{d z^{2}}=\mathbf{Z} \cdot \mathbf{Y} \vec{V}
$$

Assuming a traveling-wave solution of the form $V=V_{0} e^{r z}$, the following dispersion relation is obtained:

$$
\left|\begin{array}{cc}
\gamma^{2}-y_{d} z_{d}-y_{m} z_{m} & -\left(y_{m}+g_{m}\right) z_{d}-y_{g} z_{m} \\
-y_{m} z_{g}-y_{d} z_{m} & \gamma^{2}-y_{g} z_{g}-\left(y_{m}+g_{m}\right) z_{m}
\end{array}\right|=0 .
$$

Solving this relationship for $\gamma$ gives

in which

$$
\gamma= \pm \sqrt{\frac{B \pm \sqrt{B^{2}-4 C}}{2}},
$$

and

$$
B=y_{d} z_{d}+\left(g_{m}+2 y_{m}\right) z_{m}+y_{g} z_{g}
$$

$$
C=\left(y_{m}\left(g_{m}+y_{m}\right)-y_{g} y_{d}\right)\left(z_{m}{ }^{2}-z_{d} z_{g}\right) \text {. }
$$

This solution indicates that 4 modes exist along the device. Defining $\gamma_{1}$ as the propagation constant associated with the positive sign under the radical and $\gamma_{2}$ as the negative, the general solution along the device is

$$
V_{n}=A_{n} \mathrm{e}^{-\gamma_{1} z}+B_{n} \mathrm{e}^{\gamma_{1} z}+C_{n} \mathrm{e}^{-\gamma_{2} z}+D_{n} \mathrm{e}^{\gamma_{2} z},
$$

where $n$ represents the two electrodes ( $d$ and $g$ ). Substituting Eq. (23) into (18) gives the following relationships:

$$
\begin{aligned}
& \frac{A_{d}}{A_{g}}=\frac{B_{d}}{B_{g}}=\frac{\gamma_{1}^{2}-y_{g} z_{g}-\left(y_{m}+g_{m}\right) z_{m}}{y_{m} z_{g}+y_{d} z_{m}}=\frac{\left(y_{m}+g_{m}\right) z_{d}+y_{g} z_{m}}{\gamma_{1}^{2}-y_{d} z_{d}-y_{m} z_{m}}, \\
& \frac{C_{d}}{C_{g}}=\frac{D_{d}}{D_{g}}=\frac{\gamma_{2}^{2}-y_{g} z_{g}-\left(y_{m}+g_{m}\right) z_{m}}{y_{m} z_{g}+y_{d} z_{m}}=\frac{\left(y_{m}+g_{m}\right) z_{d}+y_{g} z_{m}}{\gamma_{2}^{2}-y_{d} z_{d}-y_{m} z_{m}} .
\end{aligned}
$$

Therefore, Eq (23) actually contains only 4 unknowns determined by the device boundary conditions (terminations) allowing for a unique solution of the device's small-signal parameters. 
The DFET, as described to this point, is a four-port device. This number of ports may be useful aspect of a DFET, but present applications generally require only two-ports. We will assume that the input port is taken to be one end of the gate electrode and the output to be the drain electrode at the opposite end of the device. The two remaining ports are terminated in $Z_{g}$ and $Z_{d}$. Analytically,

$$
\frac{V_{g}(l)}{I_{g}(l)}=-Z_{g}
$$

and

$$
\frac{V_{d}(0)}{I_{d}(0)}=Z_{d} .
$$

The two-port S-parameters are desired. Since the formulation to this point use voltages and currents, it is easy to first determine the DFET's Yparameters and then convert to S-parameters. From the definition of $Y$ parameters, we need to solve the following:

$$
\begin{aligned}
& y_{11}=\frac{I_{g}(0)}{V_{g}(0)} \mid V_{d}(l)=0, \\
& y_{21}=\frac{-I_{d}(l)}{V_{g}(0)} \mid V_{d}(l)=0, \\
& y_{12}=\frac{I_{g}(0)}{V_{d}(l)} \mid V_{g}(0)=0,
\end{aligned}
$$

and

$$
y_{22}=\frac{-I_{d}(l)}{V_{d}(l)} \mid V_{g}(0)=0
$$

where the device length is $l$. The two sets of four boundary conditions specified in (26) and (27) along with equations (11), and (23)-(25) allow for the determination of the unknown coefficients in Eq. (23). Then,

where 1 is the identity matrix.

$$
S=(1+Y)^{-1}(1-Y)
$$

The R, L,G, and $\mathbf{C}$ matrices were found using a quasi-static 2-D simulator from Ansoft as discussed earlier in this report. The simulator allows for multiple conductors and directly gives the required matrix elements shown in Table 2 for 
a $8 \times 120$ DFET assumed biased in a passive configuration. Fig. 6 shows contour plots from the electro- and magneto- static simulations.

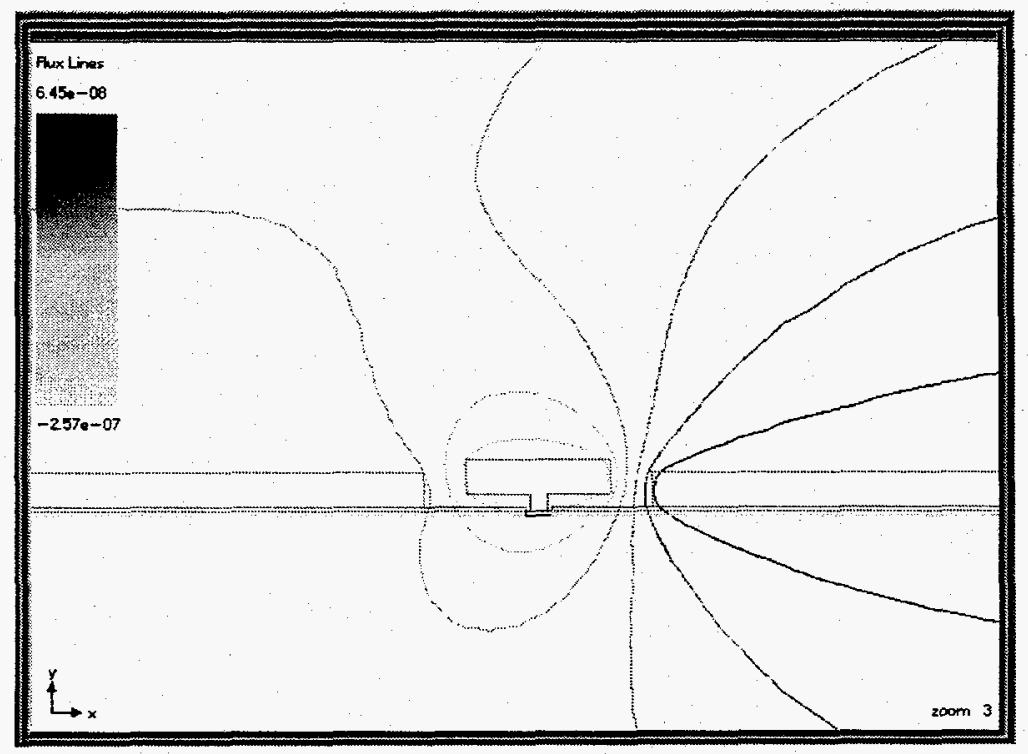

(a) Contours of longitudinal magnetic vector potential. Used in determining the impedance matrix of the DFET.

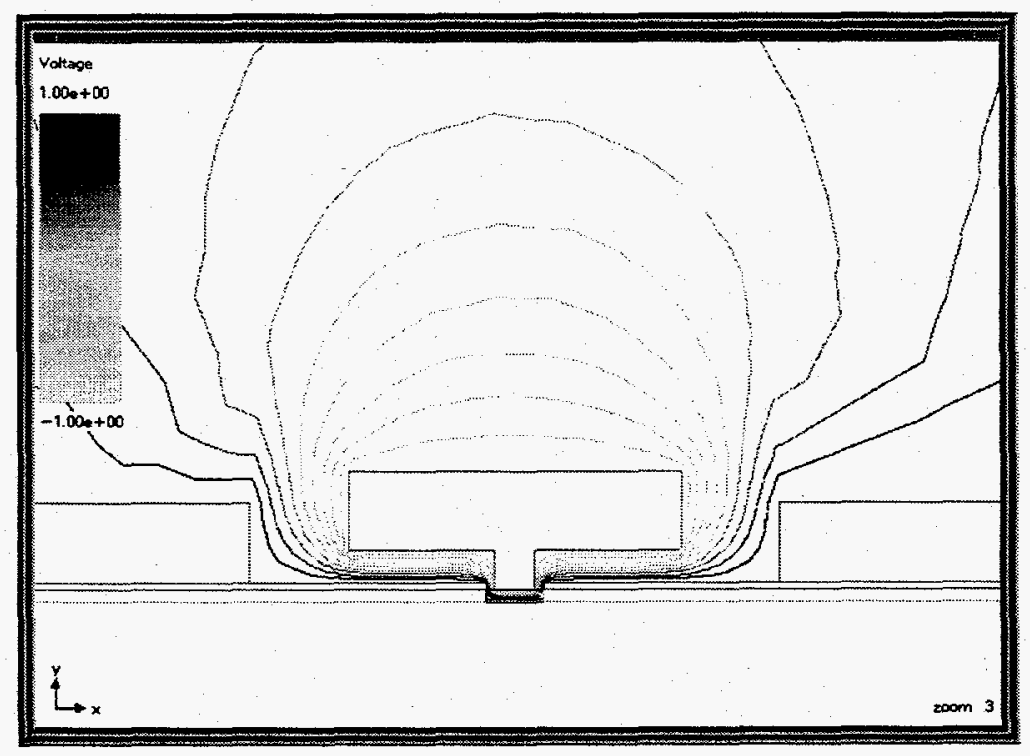

(b) Contours of constant electrostatic potential. Used in determining the admittance matrix of the DFET.

Fig. 6. Field plots from electromagnetic simulations of the DFET.

Since the matrices (13) and (14) were calculated with disregard for the device's active properties, one should expect large errors. In particular, it has 
been found that $g_{d}$ is best set to $1 / R_{d s}$ since $R_{d s}$ is a strong function of active device properties. Simulation results using this approximation are presented along with the experimental results below.

\begin{tabular}{c|cc}
\hline & Drain & Gate \\
\hline Drain & 428.7 & 7.36 \\
Gate & 7.36 & 3944.9 \\
\hline
\end{tabular}

(a) Series resistance matrix, $\mathbf{R},(\Omega / \mathrm{m})$.

\begin{tabular}{c|cc}
\hline & Drain & Gate \\
\hline Drain & $7.716 \times 10^{-7}$ & $4.288 \times 10^{-7}$ \\
Gate & $4.288 \times 10^{-7}$ & $5.366 \times 10^{-7}$ \\
\hline
\end{tabular}

(b) Series inductance matrix, L, $(\mathrm{H} / \mathrm{m})$.

\begin{tabular}{c|cc}
\hline & Drain & Gate \\
\hline Drain & 100.5 & $-3.570 \times 10^{-2}$ \\
Gate & $-3.570 \times 10^{-2}$ & 1.460 \\
\hline
\end{tabular}

(c) Conductance matrix, G, (Mho/m).

\begin{tabular}{c|cc}
\hline & Drain & Gate \\
\hline Drain & $5.651 \times 10^{-10}$ & $-3.570 \times 10^{-10}$ \\
Gate & $-3.570 \times 10^{-10}$ & $7.187 \times 10^{-10}$ \\
\hline
\end{tabular}

(d) Capacitance matrix, C, (F/m).

\section{Table 2. Calculated admittance and impedance matrix} elements at $10 \mathrm{GHz}$.

\section{DFET Fabrication}

Fig. 7 shows the DFET process sequence. The process begins with the material described in Table 3. A mesa is formed by a traditional $1 \mathrm{H}_{3} \mathrm{PO}_{4}: 4 \mathrm{H}_{2} \mathrm{O}_{2}: 45 \mathrm{DI}$ wet chemical etch and GeAu/Ni/Au ohmic contacts are formed using standard lift-off technique. The gate recess etch is defined by a thick photoresist which also defines the T-gate's base. The recess was performed with the same etchant as described above to a depth of approximately $2500 \AA$. Complications with this recess etch arose due to an electro-chemical effect caused by the large source and drain contacts exposed to the etchant. This was solved by applying a thin insulating nitride layer over the source and drain ohmic contact metals prior to etching. 
(a) Mesa is formed by a wet etch and Ohmic contacts are formed by lift-off methods.

Gate Recess PR

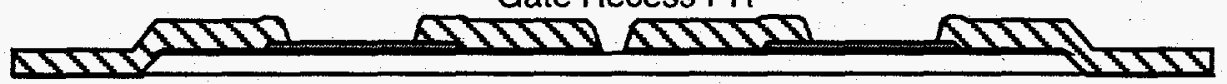

(b) $1 \mu \mathrm{m}$ thick PR is used to define gate recesses and provide support for the T-gate.

Gate/Seed Metal

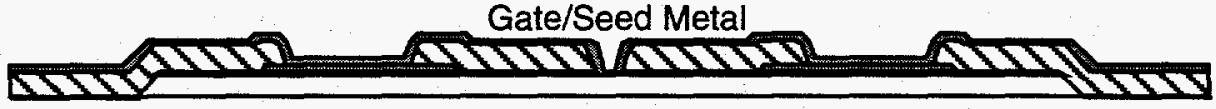

(c) Gate is defined by a seed layer.

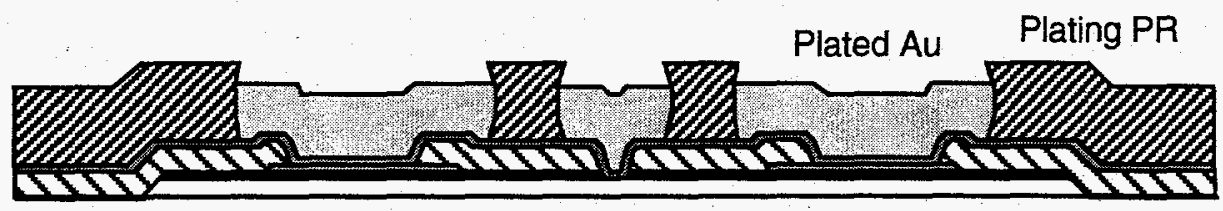

(d) Gate, source and drain are simultaneously plated with $2 \mu \mathrm{m}$ of Au.

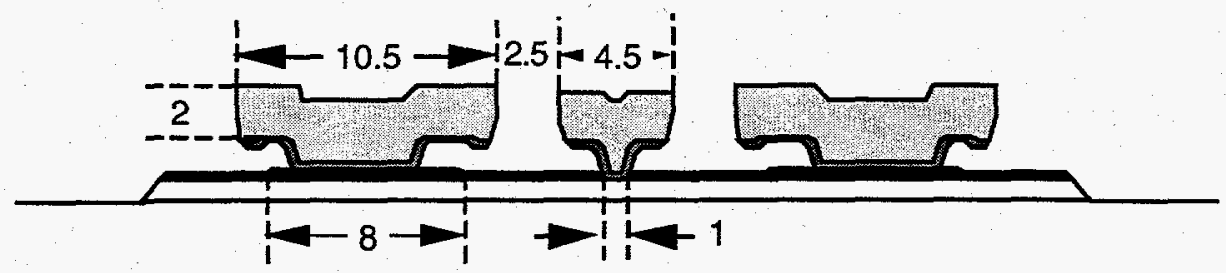

(e) Final device after PR and seed layer removal.

Fig. 7. DFET process sequence. Dimensions of final device are in micrometers.

\begin{tabular}{c|ccc}
\hline Name & Thickness & Doping Type & Doping Level \\
\hline cap & $2000 \AA$ & n-type (Si) & $5 \times 10^{18}$ \\
channel & $3200 \AA$ & n-type (Si) & $5 \times 10^{18}$ \\
buffer & $5000 \AA$ & UID & - \\
substrate & $450 \mu \mathrm{m}$ & SI & - \\
\hline
\end{tabular}

Table 3. DFET material growth specification. UID and SI mean unintentionally doped and semi-insulating, respectively.

A $3500 \AA$ thick TiAu gate contact metal forms the seed layer for a $2 \mu \mathrm{m}$ thick Au electroplated layer. This thick layer is required for low electrical propagation loss. Finally, the plating resist is removed with an oxygen plasma etch, the seed 
layer by ion milling, and the lower photoresist layer by a second oxygen plasma etch. The cross section shown in Fig. 7e results. Dimensions shown are for the $4 \times 10$ device. Fig. 8 shows SEM micrographs of a completed device.

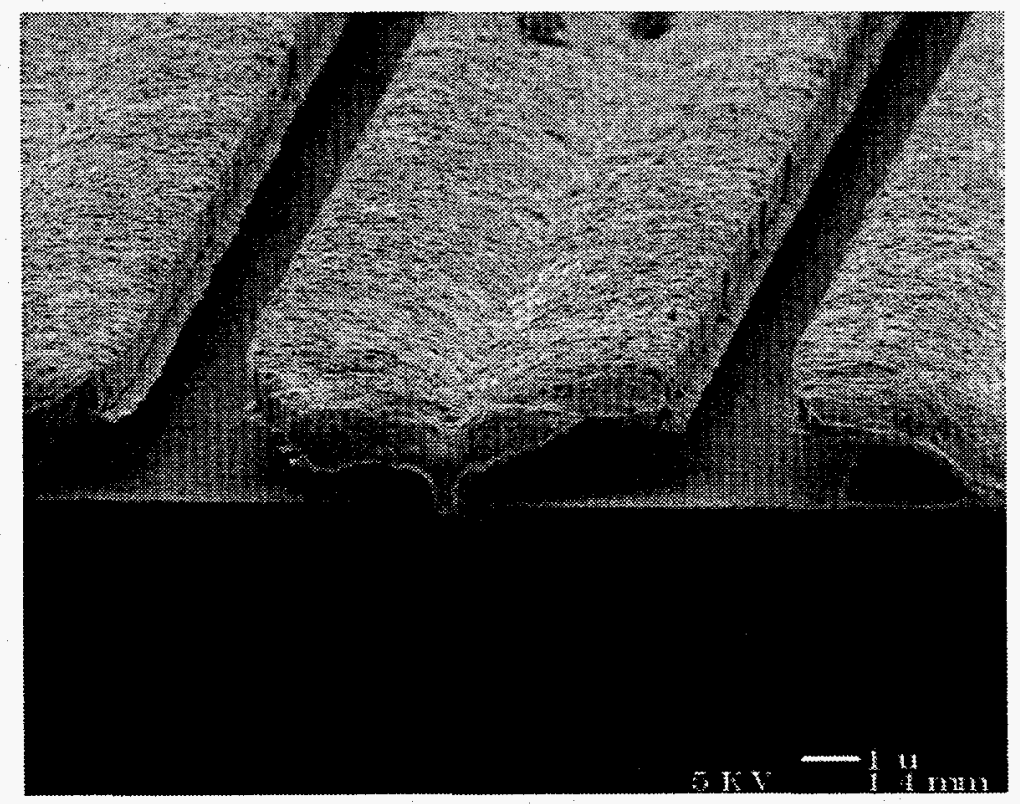

(a) Top of T-gate is $8 \mu \mathrm{m}$ across.

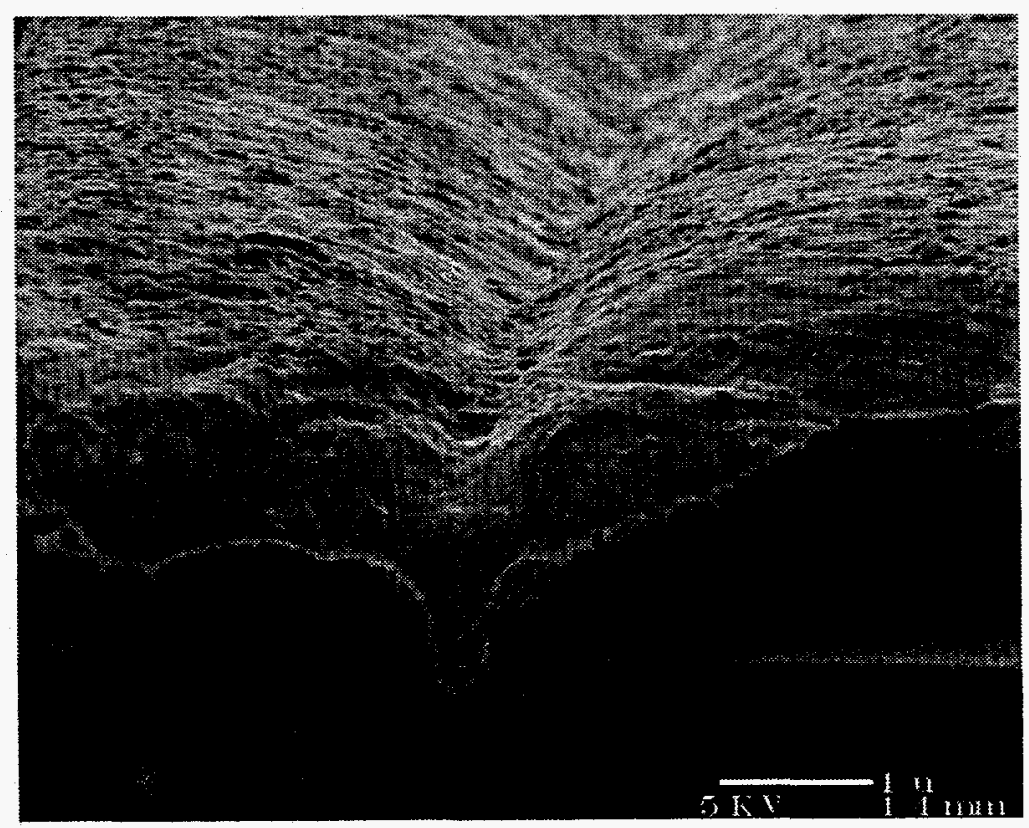

(b) Gate close-up.

Fig. 8. SEM micrographs of the DFET. 


\section{DFET Characterization}

Extensive DC and RF characterization of the DFET was performed. Results from $8 \times 120$ devices only will be presented. DC characteristics indicate good MESFET operation as shown in Fig. 9. Reasonable saturated drain currents $(>300 \mathrm{~mA} / \mathrm{mm})$ and transconductances $(>100 \mathrm{mS} / \mathrm{mm})$ were typical.

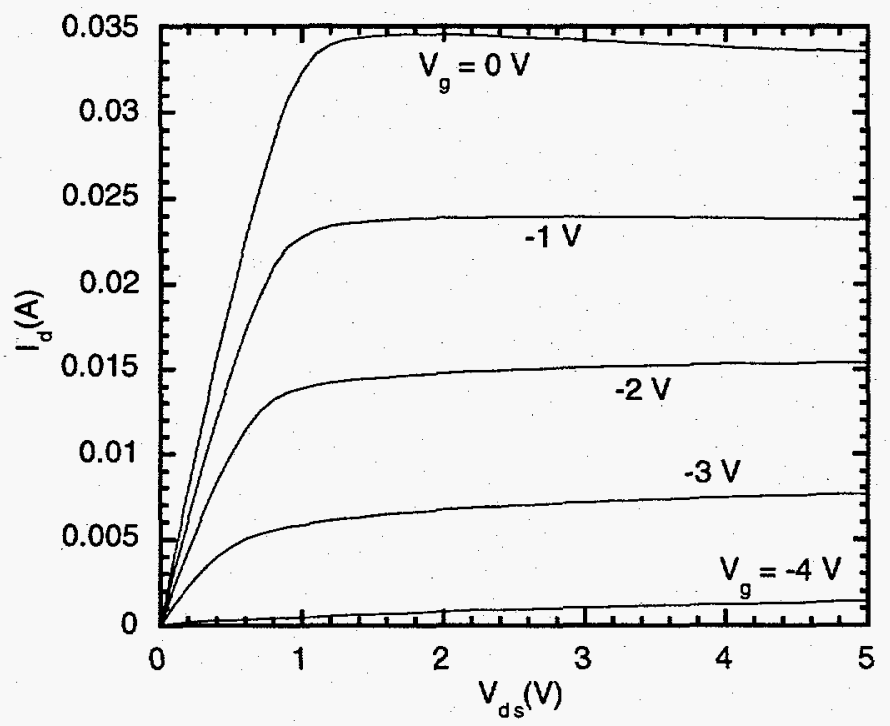

(a) I-V of a typical $8 \times 120$ DFET. Gate width is $100 \mu \mathrm{m}$.

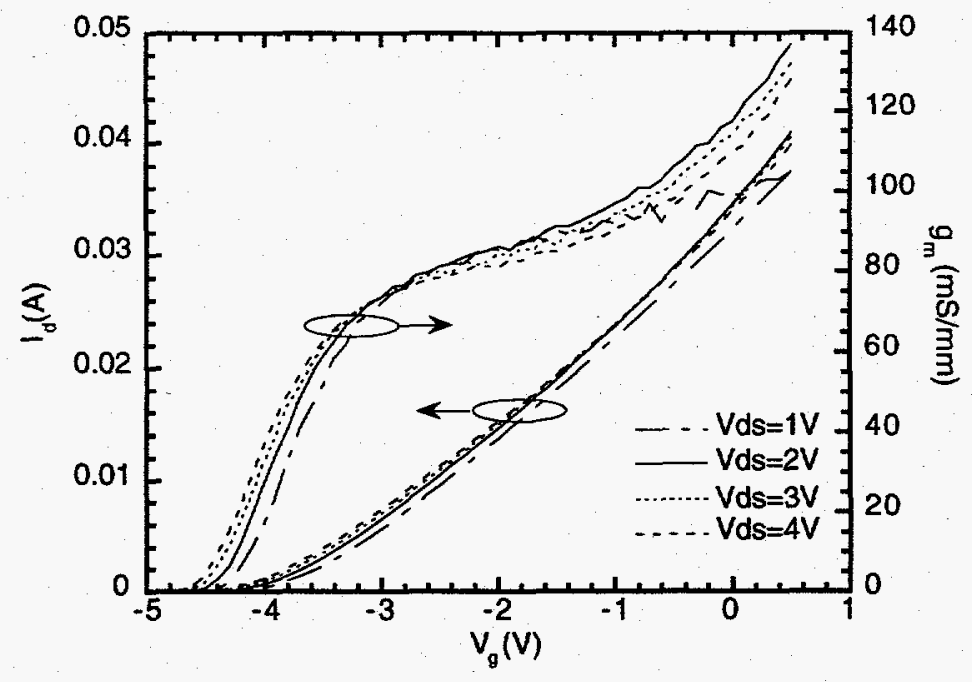

(b) $I_{d}$ and $g_{m}$ of a typical $8 \times 120$ DFET.

Fig. 9. DC electrical characteristics of a lumped $8 \times 120$ DFET (100 $\mu \mathrm{m}$ gate width).

The S-parameters from a $100 \mu \mathrm{m}$ wide $8 \times 120$ DFET test device measured from 0.05 to $26.05 \mathrm{GHz}$ at $\mathrm{V}_{\mathrm{ds}}=2 \mathrm{~V}, \mathrm{I}_{\mathrm{ds}}=7.84 \mathrm{~mA}$, and $\mathrm{V}_{\mathrm{gs}}=-3 \mathrm{~V}$ are shown in Fig. 10. These parameters were measured using ground-signal probes due to the geometry of the DFET that resulted in poor measurement quality at high 
frequencies and seen symptomatically by the "loops" in the parameters. "M1" markers are at $15 \mathrm{GHz}$ for a frequency reference. Ground-signal probes are known to suffer from radiation and spurious mode problems at high frequencies $(>18 \mathrm{GHz})$.

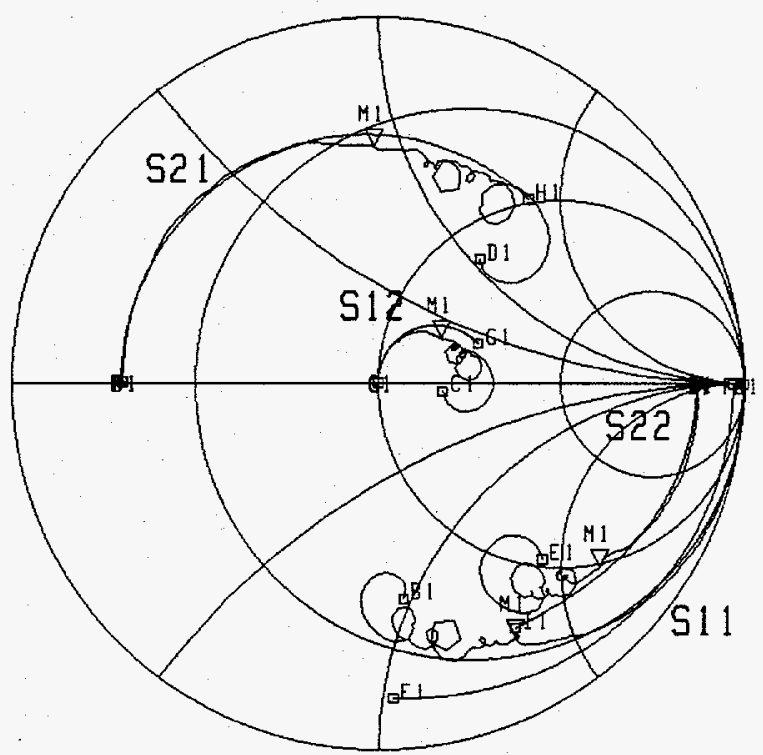

Fig. 10. Measured and modeled S-parameters of a lumped $8 \times 120$ DFET $(100 \mu \mathrm{m}$ gate width).

Fig. 11 shows the typical figure-of-merit parameters for FET's plotted against frequency. The transit time frequency, $f_{t}$, is found from the unity magnitude of $h_{21}$ which is about $\mathrm{f}_{\mathrm{t}} \approx 12 \mathrm{GHz}$ in Fig. 11. This result is not particularly impressive, but it may be consistent with the device's parasitics. Large parasitics (in a lumped sense) due to the device's large T-gate may dominate $f_{t}$ and intrinsic carrier transit times are probably much shorter than the indicated $1 / f_{\mathrm{t}}$. The unilateral transducer gain, $U$, is also plotted in Fig. 11. Again, the roughness in $U$ is due to poor measurement quality caused by the ground-signal probes. The measured $f_{\max }$ is found to be over $20 \mathrm{GHz}$ (where $|U|=1$ ) which is reasonable for a $1 \mu \mathrm{m}$ gate length MESFET.

The measured S-parameters were fit to the simple FET model shown in Fig. 12. The circuit was optimized over the frequency range of 0.05 to $15.05 \mathrm{GHz}$ to avoid the errors in the measured data occurring at high frequencies. The modeled S-parameters are plotted in Fig. 10 along with the measured data. The resulting fit parameters are shown in Table 4 along with their corresponding error estimates. The important parameters obtained from this fit are the transconductance, $7.588 \mathrm{mS}$, and output conductance, $0.001376 \mathrm{~S}$. Since the test device was $0.1 \mathrm{~mm}$ long. The transconductance and output conductance per unit length as required for the simulation are $g_{m}=75.88 \mathrm{mS} / \mathrm{mm}$ and $1 / R_{d s}=$ $G_{d s}=13.76 \mathrm{~S} / \mathrm{m}$. Note how well this value of transconductance corresponds to the DC data. 


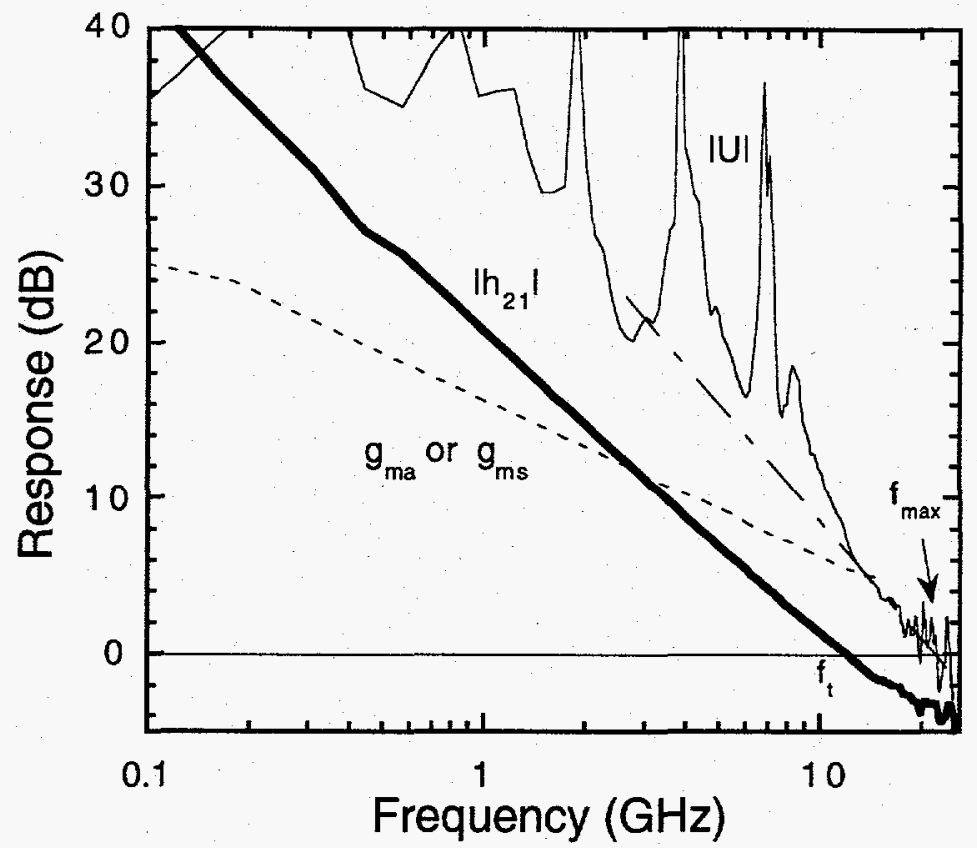

Fig. 11. Measured response of a $100 \mu \mathrm{m}$ gate width $8 \times 120$ DFET. Dash-dot line has a slope of $-20 \mathrm{~dB} / \mathrm{dec}$.

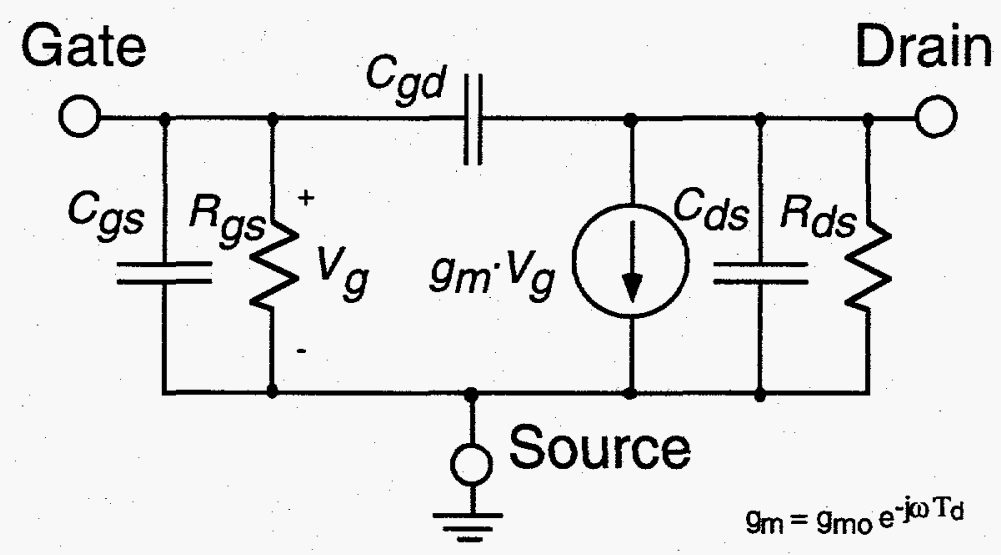

Fig. 12. Equivalent circuit of lumped DFET. 


\begin{tabular}{c|cc}
\hline Element & Value & $\begin{array}{c}\text { Error } \\
\text { Estimate }\end{array}$ \\
\hline $\mathrm{C}_{\mathrm{gs}}$ & $82.60 \mathrm{fF}$ & $\pm 0.56 \mathrm{fF}$ \\
$\mathrm{R}_{\mathrm{gs}}$ & $3443 \Omega$ & $\pm 221 \Omega$ \\
$\mathrm{C}_{\mathrm{gd}}$ & $32.67 \mathrm{fF}$ & $\pm 0.35 \mathrm{fF}$ \\
$\mathrm{g}_{\mathrm{mo}}$ & $7.588 \mathrm{mS}$ & $\pm 0.022 \mathrm{mS}$ \\
$\mathrm{T}_{\mathrm{d}}$ & $4.326 \mathrm{pS}$ & $\pm 0.068 \mathrm{pS}$ \\
$\mathrm{R}_{\mathrm{ds}}$ & $726.7 \Omega$ & $\pm 10.45 \Omega$ \\
$\mathrm{C}_{\mathrm{ds}}$ & $40.18 \mathrm{fF}$ & $\pm 0.056 \mathrm{fF}$ \\
\hline
\end{tabular}

Table 4. Lumped DFET parameters fit $(0.05$ to $15 \mathrm{GHz})$ to the equivalent circuit shown in Fig. 12.

Measurements were performed on a $2 \mathrm{~mm}$ long $8 \times 120$ DFET with the results summarized in Fig. 13 and Fig. 14. The device was biased at $V_{d s}=2 \mathrm{~V}$, $I_{\mathrm{ds}} \approx 1.5 \mathrm{~A}, \mathrm{~V}_{\mathrm{gs}}=-3 \mathrm{~V}$. In this measurement ground-signal probes were used again with one probe taking the input from gate to source and the other probe taking the output on the opposite end of the device from drain to source. The remaining "ports" were not terminated $(Z \rightarrow \infty)$. The results from the theoretical model described earlier, are also plotted in Fig. 13 and Fig. 14 as solid lines. Both plots show a surprisingly good correspondence and certainly the theory shows the correct qualitative response. Recall that the element matrices were only calculated at $10 \mathrm{GHz}$ and these elements are known to be somewhat frequency dependent -- especially at low frequencies.

The gains shown in Fig. 13 and Fig. 14 are not particularly large, but they do demonstrate distributed operation. Notice that the magnitude of $U$ is not much larger than the magnitude of $S_{21}$ indicating further impedance matching would not significantly improve device performance. This is one of the predicted benefits of a distributed device. Also, note that the device exhibits gain at frequencies well over the traditional transit time cutoff. Again, this is expected for a distributed transistor as device parasitics are "absorbed" into the transmission line. Higher gains would be expected with longer devices, though measurements performed on longer devices $(5 \mathrm{~mm})$ resulted in similar results as in Fig. 13 and Fig. 14. Additional studies of the internal wave configurations of the device will hopefully yield a more optimal device design or termination conditions to allow for higher-speed and higher-gain operation. 


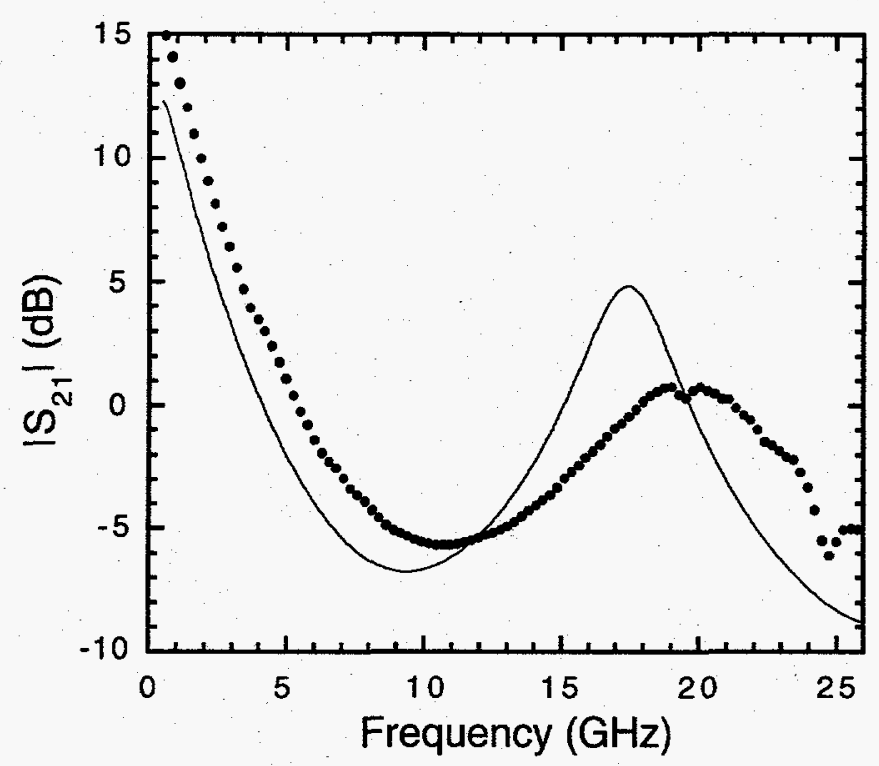

Fig. 13. Comparison between measured (points) and theoretical (line) magnitude of $S_{21}$.

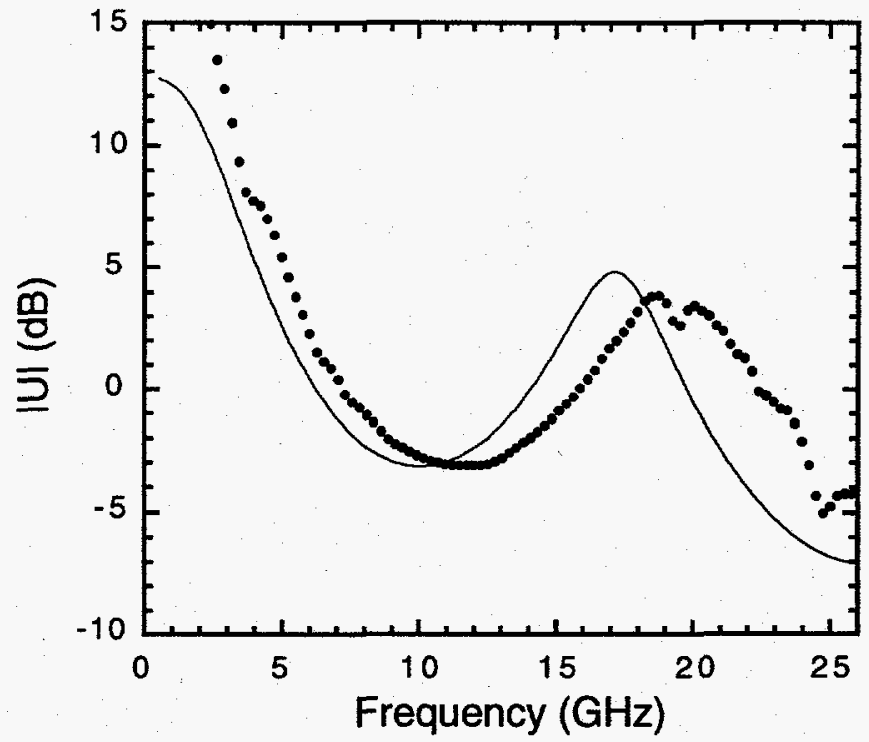

Fig. 14. Comparison between measured (points) and theoretical (line) magnitude of the unilateral transducer gain, IUI. 


\section{Conclusions}

The importance of distributed device design for ultra-high-speed operation was shown and results from both distributed optical and electronic devices were summarized in this report. Distributed electrodes in optoelectronic devices allow for operation at frequencies well within the millimeter-wave frequency range. Similarly, future work should allow for distributed electronic devices to operate at millimeter wave frequencies at greatly increased power levels and efficiencies as compared to present-day lumped designs. 


\section{References}

[1] H. Hasegawa, and H. Okizaki, "M.I.S. and Schottky slow-wave coplanar striplines on GaAs substrates," Electronic Letters, Vol. 13, No. 22, pp. 663-664, Oct. 1977.

[2] T. Fukuoka, and T. Itoh, "Analysis of slow-wave phenomena in coplanar waveguide on a semiconductor substrate," Electronic Letters, Vol. 18, No. 14, pp. 589-590, July 1982.

[3] Y. Fukuoka, Y. Shin, and T. Itoh, "Analysis of slow-wave coplanar waveguide for monolithic integrated circuits," IEEE Trans. Microwave Theory and Tech., Vol. 31, pp. 567-573, 1983.

[4] Y. R. Kwon, V. M. Hietala, and K. S. Champlin, "Quasi-TEM analysis of slow-wave mode propagation on coplanar microstructure MIS transmission lines," IEEE Tans. Microwave Theory Tech., Vol. 35, pp. 545-551, 1987.

[5] Maxwell 2-D, Ansoft Corporation, Pittsburgh, PA 15219.

[6] J. G. Meddoza-Alverez, L. A. Coldren, A. Alping, R. H. Yan, T. Hausken, K. Lee, and K. Pedrotti "Analysis of Depletion Edge Translation Lightwave Modulators," IEEE J. Lightwave Tech., vol. 6, no. 6, pp. 793-808, June 1988.

[7] J. R. Pierce and L. M. Field, "Traveling-wave tubes," Proc. IRE, vol. 35, pp. 108-111, Feb. 1947.

[8] L. Solymar, and E. A. Ash, "Some Travelling-wave Interactions in Semiconductors Theory and Design Considerations," Int. J. Electronics, Vol. 20, No. 2, pp. 127$148,1966$.

[9] R. H. Dean, and R. J. Matarese, "The GaAs Traveling-Wave Amplifier as a New Kind of Microwave Transistor," Proceedings of the IEEE, Vol. 60, No. 12, pp. 14861502, 1972.

[10] G. W. Mclver, "A Traveling-Wave Transistor," Proceedings of the IEEE, pp. 17471748, Nov. 1965.

[11] A. S. Podgorski, and L. Y. Wei, "Theory of Traveling-Wave Transistors," IEEE Transactions on Electron Devices, Vol. ED-29, No. 12, pp. 1845-1853, December 1982.

[12] A. J. Holden, D. R. Daniel, I. Davies, C. H. Oxley, and H. D. Rees, "Gallium Arsenide Traveling-Wave Field-Effect Transistors," IEEE Transactions of Electron Devices, Vol. ED-32, No. 1, pp. 61-66, Jan. 1985.

[13] W. Heinrich, and H. L. Hartnagel, "Wave Propagation on MESFET Electrodes and Its Influence on Transistor Gain," IEEE Trans. on Microwave Theory and Techniques, Vol. MTT-35, No. 1, pp. 1-8, 1987.

[14] K. Fricke, V. Krozer, H. L. Hartnagel, "A New GaAs Power MESFET Structure for Improved Power Capabilities," IEEE Trans. on Microwave Theory and Techniques, Vol. 37, No. 9, pp. 1334-1339, Sept. 1989. 
[15] N. Sebati, P. Gamand, C. Varin, F. Pasqualini, and J.-C. Meunier, "Continuous Active T-Gate Travelling-Wave Transistor," Electronics Letters, Vol. 25, No. 6, pp. 403404, March 1989.

[16] T. T. Y. Wong, Fundamentals of Distributed Amplifications, Chapter 5, pp. 115-163, Artech House, Inc., Norwood, MA, 1993. 


\title{
Appendix A. Reprint of "High-performance GaAs/AlGaAs optical phase modulators for microwave/photonic integrated circuits"
}

Hietala, V. M., S. H. Kravitz, M. G. Armendariz, G. A. Vawter, R. F. Carson, and R. E. Leibenguth, 1994. "High-performance GaAs/AIGaAs optical phase modulators for microwave/photonic integrated circuits," presented at Optoelectronic Signal Processing for Phased Array Antennas IV, SPIE OE LASE '94, Los Angeles, CA, 22-28 January.

\section{High-performance GaAs/AIGaAs optical phase modulators for microwave/photonic integrated circuits}

\author{
V. M. Hietala, S. H Kravitz, M. G. Armendariz, G. A. Vawter, \\ and R. F. Carson
}

Sandia National Laboratories, Albuquerque, NM 87185-0603

R. E. Leibenguth

AT\&T Bell Laboratories, Breinigsville, PA 18031

\begin{abstract}
High-speed high-performance optical phase modulators are being developed for use in a coherent Photonic Integrated Circuit (PIC) technology. These phase modulators are the critical component of a PIC program at Sandia National Laboratories targeted for microwave/millimeter-wave signal processing and control including phased-array antenna control. The primary design goals for these modulators are amenability for integration into PICs, high "figure of merit" (FOM - phase shift per unit length-voltage), and large bandwidths allowing for operation at millimeter wave frequencies. Depletionedge-translation optical phase modulators (GaAs/AlGaAs based) have been selected as the device technology of choice due to their high FOM $(>60 \% / \mathrm{N} \cdot \mathrm{mm}$ @ $1.3 \mu \mathrm{m})$. These modulators unfortunately suffer from a large terminal capacitance which greatly limits speed. To overcome this problem, a distributed electrode design based on the use of slow-wave coplanar strips has been developed. Device design and measurements are presented in this paper.
\end{abstract}




\section{MOTIVATION}

High performance optical phase modulators for photonic integrated circuit (PIC) applications must not only be high-speed but also physically small. This can be appreciated by inspecting the symbolic layout of COMPASS shown in Figure 1. COMPASS (Coherent Optical Monolithic Phased Array Steering System) is an internal program at Sandia which drives many of our PIC efforts. This proposed system, which has been discussed in detail elsewhere, [1] is an ideal example of the type of complexity anticipated in PIC's based entirely on optical phase shifters.[2] COMPASS has several optical phase modulators in every signal path from input to output. Specifically, in the optical frequency translator portion of the circuit alone, the total peak phase shift required is 8.4 radians or approximately $480^{\circ}$ of phase shift. Assuming a reasonable bias level of $5 \mathrm{~V}$, circuit size can easily become prohibitively large. If a given phase modulator had a meager FOM of $4.5 \% / \mathrm{N} \cdot \mathrm{mm}$ (as with some high-speed optical phase modulators[3]), the optical frequency translator alone would need be $2.1 \mathrm{~cm}$ in length -- a length not realistic for a semiconductor circuit. Using instead a modulator with a FOM of $60 \% / \mathrm{Nm}$ as typical of the modulators discussed here, the total length would be only $0.16 \mathrm{~cm}$. In practice significant additional lengths might be required for the necessary power splitters and combiners for the circuit, but total circuit length will still be dominated by phase shifter length.

This example shows that a high FOM phase modulator is essential for producing complex PICs. Certainly, a high FOM will have a very significant impact on device yield. To obtain this necessary high FOM, depletion-edgetranslation optical phase modulators (GaAs/AIGaAs) have been selected as the device technology of choice for our PIC effort. 
a)
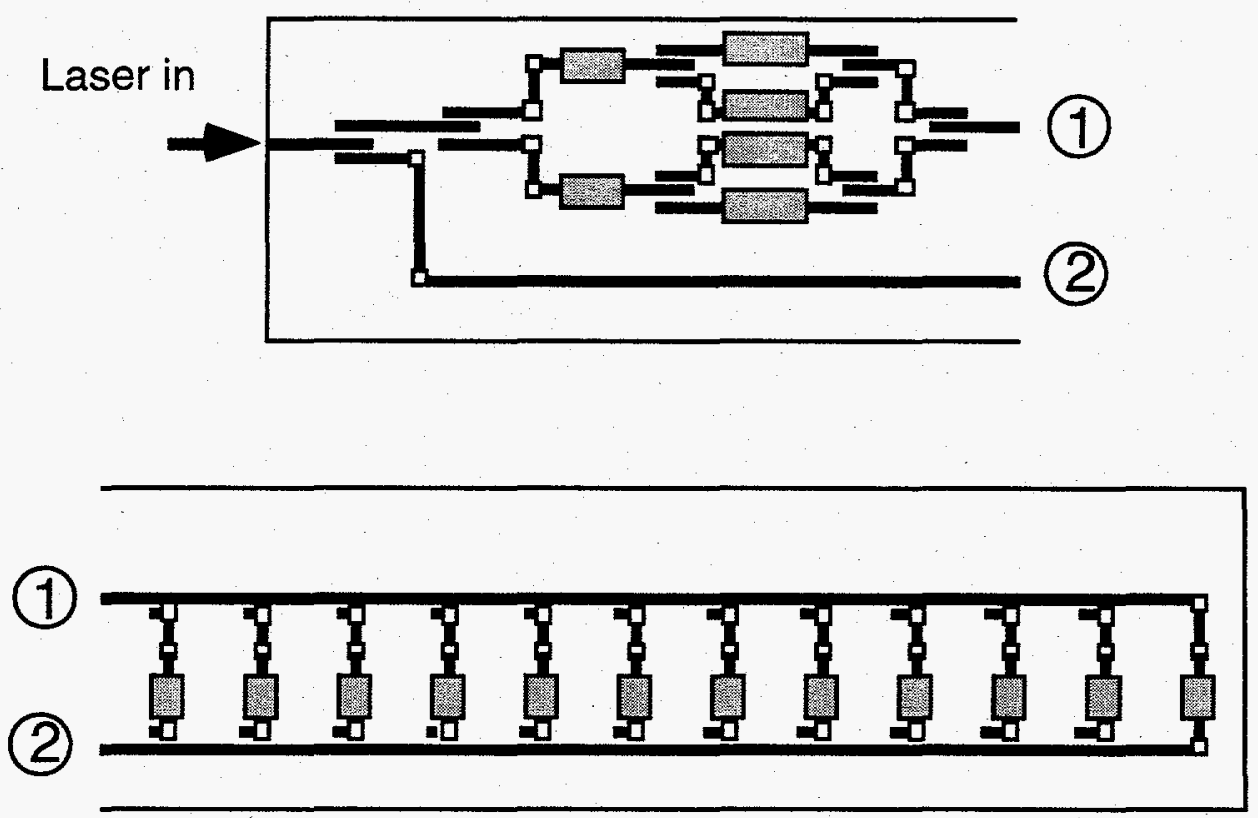

b)

Optical Waveguide

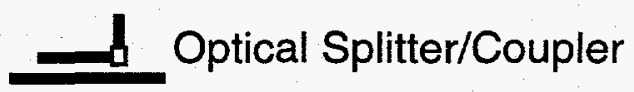

Optical Phase Shifters

D Turning Mirrors

日 Normal Turning Mirrors

Fig. 1. Symbolic layout of COMPASS. a). Optical frequency translator. b). Output power dividers and phase control.

\section{DEPLETION-EDGE-TRANSLATION PHASE MODULATOR}

Figure 2 shows details of the depletion-edge-translation phase modulator. This and similar designs have been discussed in detail in the literature.[4] Based on the basic design outlined in Fig. 2, we have observed phase shifts as large as $64 \% \mathrm{~N} \cdot \mathrm{mm}$ at $1.3 \mu \mathrm{m}$ with optical losses around $4 \mathrm{~dB} / \mathrm{cm}$. Fabrication details of this structure can be found in a separate paper.[5] Unfortunately, these depletion-edge-translation optical phase modulators suffer from large device capacitance which greatly limits device speed if conventional lumped designs are used. This large capacitance occurs due to the heavy doping required to produce large electric fields within the modulator for high FOM operation. Lumped depletion-edge-translation modulators in our laboratory have exhibited experimental bandwidths of only about $1 \mathrm{GHz}--$ a speed inadequate for all but the slowest of microwave applications. To obtain high-speed operation, a distributed electrode design based on the use of slowwave coplanar transmission lines has been developed. 


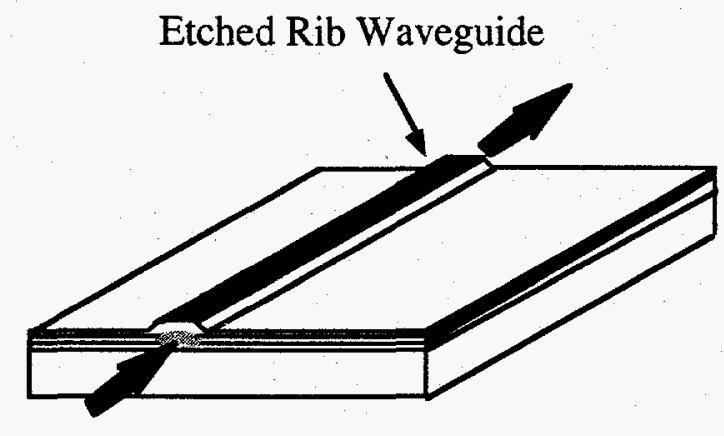

a)

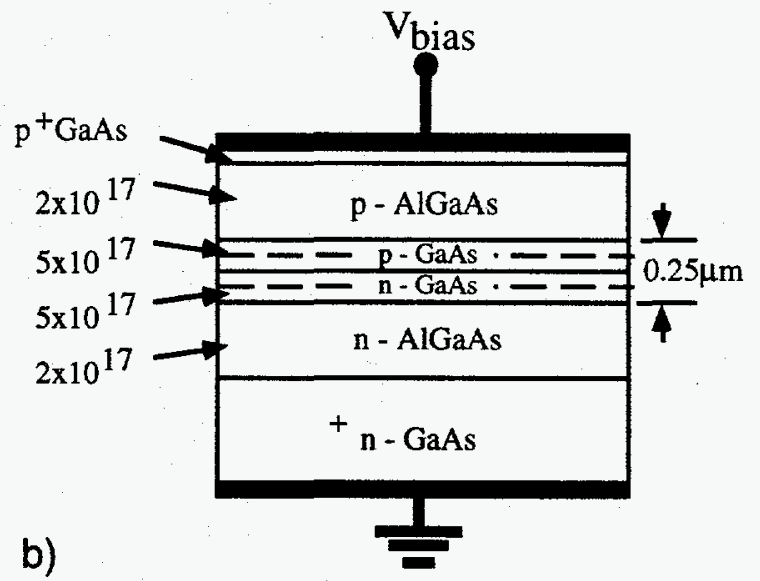

Fig. 2. Reverse-biased depletion-edge-translation optical phase modulator. a) Rib waveguide design. b) Cross section of modulator region. Dashed lines represent depletion layer edges. Center GaAs layers provide index change for light guiding.

\section{ELECTRODE DESIGN}

In order to produce a high-speed depletion-edge-translation optical phase modulator it is necessary to provide impedance control and a velocity match to the degree sufficient to avoid "phase cancellation" along the device's length. Impedance control is necessary to provide an optical power match from the drive electronics to the modulator. As a minimum, it is desirable for the modulator to have a frequency-independent real load impedance. This is generally not difficult, if the characteristic impedance of the device is known, and a resistive termination is used.

Avoiding "phase cancellation" is a more complex problem. In general, optical phase modulators are large, due to the weak interaction between light and the modulating media. Due to this extended length of interaction with the light, velocity synchronism of the light to the electrical drive signal along the length of the modulator becomes important. In the worst case, the electrical drive signal can become exactly $180^{\circ}$ out of phase with the modulated light along the device length. This will occur periodically with frequency with the lowest frequency $\left(f_{r}\right)$ at :

$$
f_{r f}=\frac{c}{2 L|\Delta n|}
$$

in which $L$ is the length of the device, $c$ is the speed of light, and $\Delta n$ is the difference between the optical index $n_{0}$ and the if index $n_{r}$. This equation sets an absolute maximum frequency limit on the allowed velocity mismatch in a distributed phase modulator. Naturally, the $-3 \mathrm{~dB}$ point will be at a somewhat lower frequency, but Eq. 1 is still a good goal for design. Here, the rib guide 
structure discussed earlier has an optical index of approximately 3.3 with a FOM of approximately $60 \% / \mathrm{V} \cdot \mathrm{mm}$. Assuming a device length of $1.0 \mathrm{~mm}\left(300^{\circ}\right.$ for $5 \mathrm{~V}$ bias) and operation to $30 \mathrm{GHz}, \Delta n$ must be less than 5.0 . This is equivalent to $n_{n f}$ being between 1.0 to 8.3. This large range of acceptable if index is a result of the short length of the device due to the device's high FOM. In spite of this large index range, it is still difficult to design an appropriate modulator electrode structure.

It has long been known that planar transmission lines fabricated over doped semiconductors result in slow wave propagation.[6] Depletion-edgetranslation optical phase modulators are based on high doping levels which results in very slow electrical propagation along the device's length. Some of our first lumped modulator designs had propagation velocities of less than 1/20 the speed of light. $\left(n_{n f} \approx 20\right)$ - a speed not useful in spite of the minimal velocity-match requirements developed above. Fortunately, it is not to difficult to increase the velocity propagation to acceptable levels.

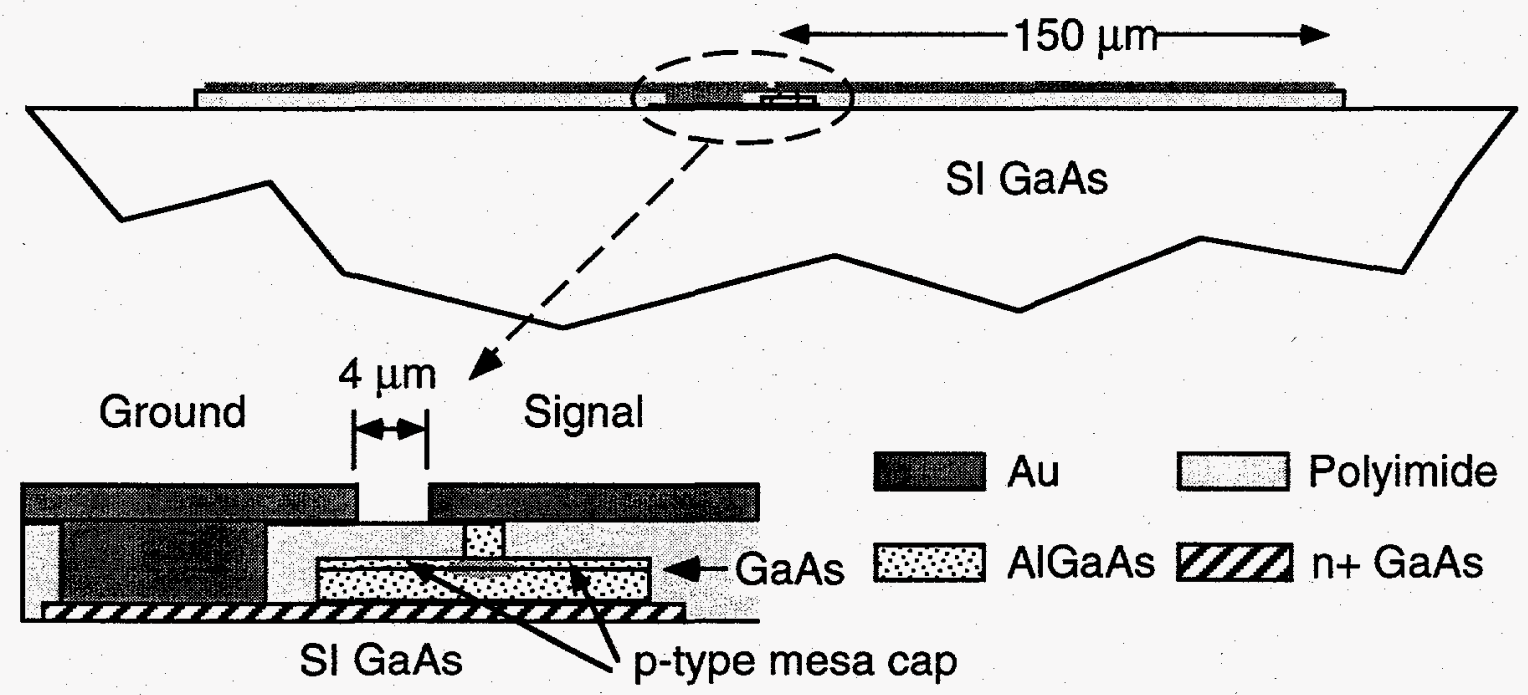

Fig. 3. Cross section of the slow-wave coplanar-strip electrode design.

Propagation along slow-wave transmission lines of the type relevant here can be described to first order by quasi-TEM analysis.[7] That is, the propagation can be adequately described by the unit length impedance $Z$ and admittance $Y$ of the transmission line. Once knowing $Z$ and $Y$, the transmission lines characteristic impedance $Z_{0}$ and propagation constant $\gamma$ can be found from:

$$
Z_{o}=\sqrt{\frac{Z}{Y}} \quad \text { and } \quad \gamma=\sqrt{Z Y}
$$

The real part of $\gamma$ gives the propagation loss along the electrode and the imaginary part gives the velocity along the electrode. The if index $\left(n_{r f}=c / v_{g}\right)$ is related to the imaginary part of $\gamma$ by $n_{r f}=d \omega \operatorname{Im}(\gamma)$ in which $c$ is the speed of 
light, and $\omega$ is the angular $r$ frequency. $Z$ and $Y$ can be estimated analytically for simple structures or calculated numerically for more complex structures. We have selected a quasi-static finite element package from Ansoft[8] to determine the admittance and impedance per unit length of our devices.

Analysis of various possible electrode structures indicated that it is very difficult to obtain a perfect velocity match between the light and electrical drive signal due to the necessary large device capacitance per unit length. This is particularly true if a characteristic impedance close to $50 \Omega$ is desired. As a first attempt, the coplanar strip-like structure shown in Fig. 3 was selected. One of the interesting features of this electrode structure is the small gap selected between the ground and signal electrodes. This requirement for a small gap may be counter-intuitive, as it increases the capacitance of the device which would be expected to further slow propagation. Though capacitance is increased, decreasing this gap also reduces the inductance per unit length so that the net effect is to increase the velocity of propagation along the line. Being able to separately adjust a transmission line's inductance and capacitance per unit length is a feature of slow-wave transmission lines. In conventional linear transmission lines the inductance and capacitance per unit length are coupled by the propagation velocity $\left(v_{g}=1 / \sqrt{L C}\right)$ which is determined by the dielectric in the transmission line.

\section{EXPERIMENTAL}

Devices were fabricated and tested both electrically for transmission line properties and optically for phase modulator performance. Fig. 4 shows measured and simulated results for the electrical transmission line properties of the modulator. These measurements were made with on-wafer microwave probes. The measured S-parameters were directly converted to the transmission line parameters shown in Fig. 4. The if index is seen to still be too large (>7); the characteristic impedance is rather low; and losses are somewhat excessive.

The propagation velocity along the device can be increased by minimizing device capacitance and inductance per unit length. Capacitance can be minimized by reducing stray capacitances and junction area (implantation). Capacitance can also be reduced by increasing junction width with some sacrifice of FOM. The inductance can be reduced by further minimizing the gap between the electrodes. The low characteristic impedance is unfortunately a requirement for a fast transmission line with large capacitance per unit length $\left(Z_{o} \approx 1 /\left(C \cdot v_{g}\right)\right)$.

Propagation losses are the primary factor limiting device speed. They can be reduced somewhat by increasing metal thickness, but a more important factor is to minimize the doped materials where the electric field is large within the structure. This was a rather surprising result discovered during simulation. The small (sub micron thickness) moderately doped p-type mesa cap to either 
side of the waveguide rib as shown in Fig. 3, constitute most of the propagation loss at microwave frequencies. The elimination of this cap is expected to at least halve the of loss along the electrodes. An electrical isolation implant is being used to effectively remove this cap in future device designs.
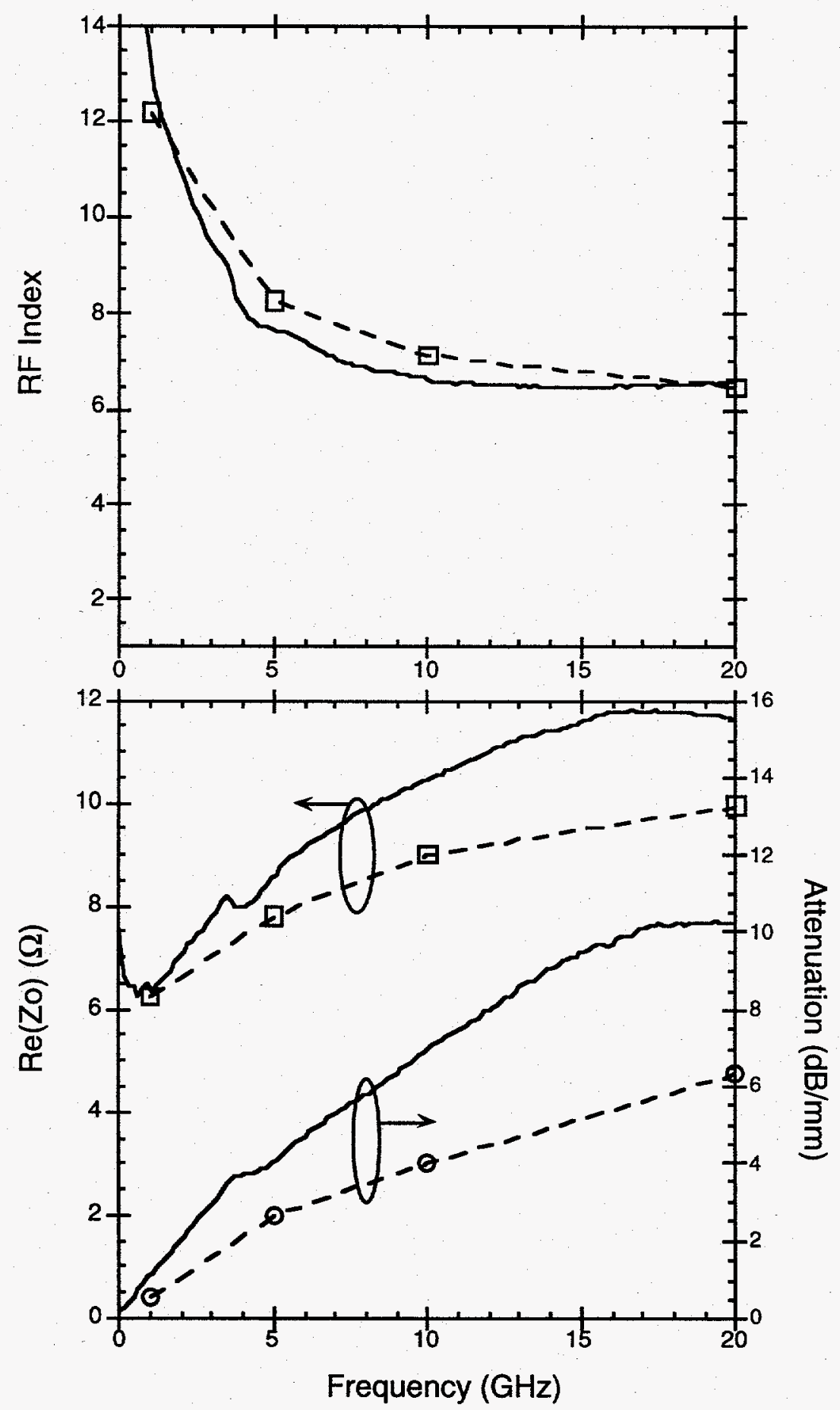

Fig. 4. Transmission line properties of modulator. Lines are measured. Symbols are theory. 
To perform high-speed testing, the modulators were packaged with optical connections made by butt-coupling to single-mode fibers. Electrical connections were made by ribbon bonding from microstrip lines. Fig. 5 shows the measured response from $0.045-20 \mathrm{GHz}$, using an HP $8510 \mathrm{C}$ network analyzer. The modulator was driven optically with a $1.3 \mu \mathrm{m} \mathrm{Nd}$ :YAG laser. Electrical drive was directly applied from one port of the network analyzer and a $35 \mathrm{GHz}$ bandwidth photodetector was used at the other port to detect the modulated light. Normalization of the response occurred during network analyzer calibration. No correction for detector response or other system losses was performed which makes the normalization in Fig. 5 arbitrany. Signal strength is seen to be somewhat low in Fig. 5. The measured noise floor, however, was in excess of $100 \mathrm{~dB}$ so the measurements should be valid. Several factors contribute to the low signal strength. The most significant signal loss was due to the large optical loss resulting from the fiber butt-coupling. Additionally, the phase modulator's facets were anti-reflection coated prior to packaging which eliminates all Fabry-Perot modes in the modulator. This means, true phase-modulation was used which is inefficient when detected by a photodetector. Finally, the measurement was performed with no impedance matching of the if source $(50 \Omega)$ to the device $(\approx 9 \Omega)$.

Several interesting features are present in the measured response. First, a large initial roll-off is evident at low frequencies. This is due to the bias network used in the experiment. The device was packaged with a $10 \Omega$ resistive termination which required a blocking capacitor in series for DC bias. This results in a theoretical $16 \mathrm{~dB}$ response roll off at low frequencies -- similar to that measured. Secondly, device response is limited to an approximate usable bandwidth of $10 \mathrm{GHz}$. This bandwidth limitation is due to device loss rather than velocity mismatch. This can be seen by observing the "counter-propagating" response which is also shown as a dotted line in Fig. 5. The counterpropagating response is seen to have a notch at $13 \mathrm{GHz}$. Applying Eq. 1 and assuming the light propagates with $n_{0}=-3.3$ (opposite the electrical drive signal), the if index must be $n_{r f}=8.2$. This $f$ index is in reasonable agreement with the measured $n_{i f}$ in Fig. 4. Again applying Eq. 1, the bandwidth for copropagating operation should be $30 \mathrm{GHz}$. This frequency is much higher than the present device bandwidth; so therefore, device speed is not presently limited by velocity mismatch. These calculations indicated that the bandwidth is limited by excessive electrical propagation loss along the device. This is particularly evident in light of the measured electrical loss in Fig. 4.

This present modulator design suffers from several disadvantages, with the most significant problem being electrical propagation loss. A new modulator design employing the changes outlined here is currently in process. The new design uses a slow-wave coplanar transmission line design for the electrodes. This will allow for a faster electrical propagation, which will virtually eliminate the velocity matching issue except for operation in excess to $100 \mathrm{GHz}$. Additionally, great care is being taken to eliminated all excess electrical losses in the device. The theoretical bandwidth of this new device is $30 \mathrm{GHz}$ with useful operation extending well within the mm-wave band. Fabrication details of this new device can be found elsewhere.[5] 


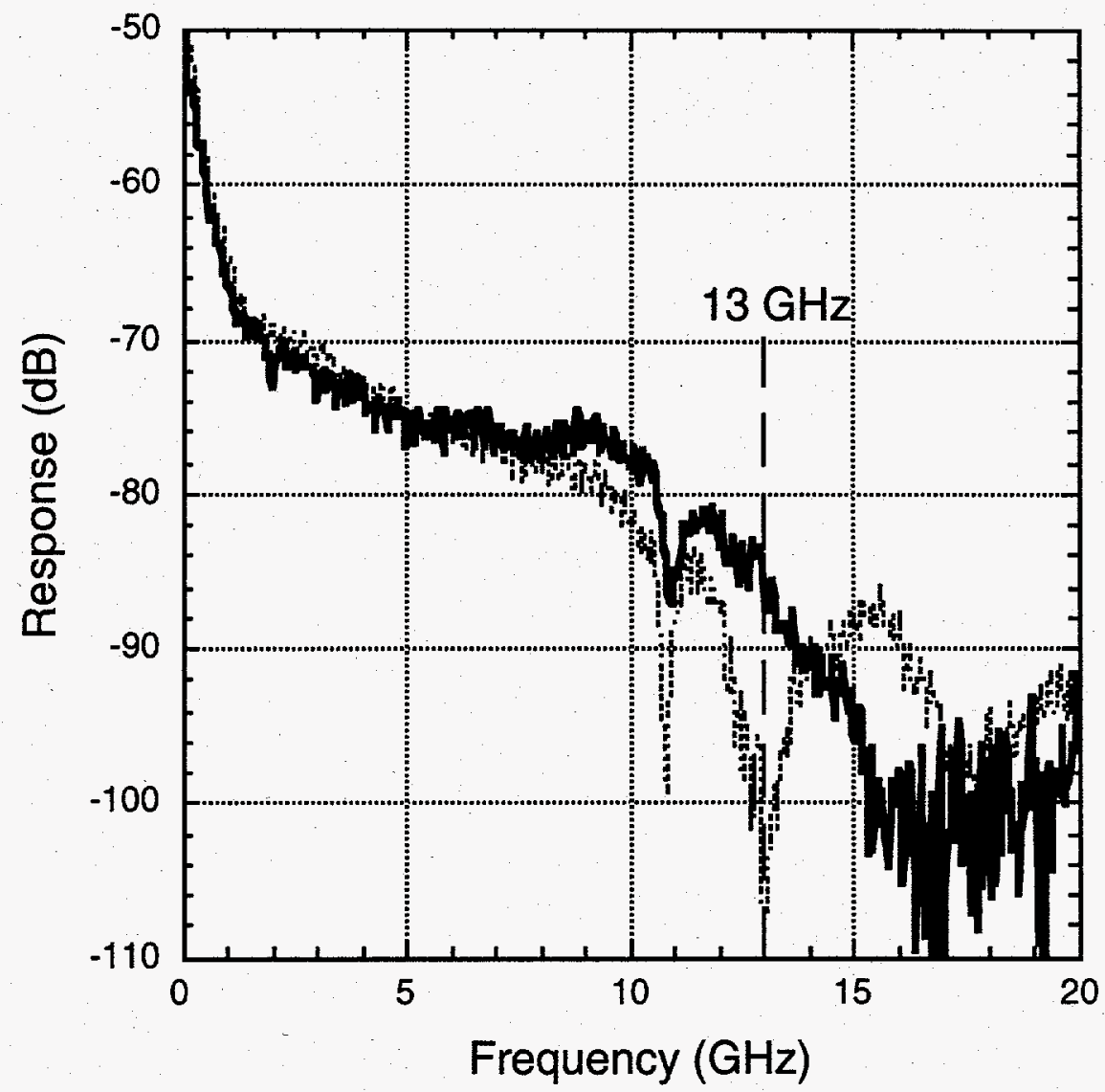

Fig. 5. Measured response of a packaged $1 \mathrm{~mm}$ length optical phase modulator. The solid line is co-propagating operation and the dashed line is counter-propagating. The notches in both responses at approximately $11 \mathrm{GHz}$ are believed to be due to a package resonance.

\section{CONCLUSIONS}

The design and test of a high-speed large-FOM optical phase modulator was presented. The current design has obtained usable operation to $10 \mathrm{GHz}$ while maintaining high FOM. Calculations indicate and measurements confirm that a velocity match in depletion-edge-translation optical phase modulators is difficult, making true traveling-wave operation unlikely. Nevertheless, the technique presented here offers the reduction of velocity mismatch and provides for a controlled drive impedance. It should be possible to produce depletion-edge-translation optical phase modulators for PIC use which can operate at millimeter-wave speeds $(>30 \mathrm{GHz}$ ) while simultaneously maintaining very high FOMs $(>600 \mathrm{~N} \cdot \mathrm{mm})$ for small circuit size. 


\section{ACKNOWLEDGMENTS}

This work was performed at Sandia National Laboratories supported by the U. S. Department of Energy under contract \#DE-AC04-94AL85000.

\section{REFERENCES}

[1]V. M. Hietala, G. A. Vawter, W. J. Meyer, and S. H Kravitz, "Phased-array antenna control by a monolithic photonic integrated circuit", Proceeding of the SP:E, Vol. 1476, Optical Technology for Microwave Applications V, pp. 170-175, April 3-5, Orlando, FL, 1991.

[2]G. A. Vawter, "Processing Issues and Technologies for Optoelectronic Integrated Circuits and Devices (Invited)," Proceeding of the SPIE, Vol. 1582, Integrated Optoelectronics for Communication and Processing, pp. 178-184, Sept. 3-4, Boston, MA, 1991.

[3]R. G. Walker, "High-Speed III-V Semiconductor Intensity Modulators", IEEE J. of Quantum Electronics, Vol. 27, No. 3, pp. 654-667, March 1991.

[4]J. G. Meddoza-Alverez, L. A. Coldren, A. Alping, R. H. Yan, T. Hausken, K. Lee, and K. Pedrotti "Analysis of Depletion Edge Translation Lightwave Modulators," IEEE J. Lightwave Tech. Vol. 6, No. 6, pp. 793-808, June 1988.

[5]S. H Kravitz, G. A. Vawter, V. M. Hietala, M. B. M. Snipes, Jr., M. G. Armendariz, R. F. Carson, B. E. Hammons, and R. E. Leibenguith, "Fabrication of high-performance GaAs/AIGaAs optical phase modulators for microwave/photonic integrated circuits", to be published in Proceeding of the SPIE, Vol. 2155-15, January 2627, 1994.

[6]H. Guckel, P. A. Brennan, and I. Palocz, "A Parallel-Plate Waveguide Approach to Microminiaturized, Planar, Transmission Lines for Integrated Circuits", IEEE Trans. Microwave Theory and Techniques, Vol. MTT-15, no. 8, pp. 468-476, August 1967.

[7]Y. R. Kwon, V. M. Hietala, and K. S. Champlin, "Quasi-TEM Analysis of "Slow-Wave" Mode Propagation on Coplanar Microstructure MIS Transmission Lines", IEEE Trans. Microwave Tech., MTT-35, June 1987.

[8]Maxwell 2-D, Ansoft Corporation, Pittsburgh, PA 15219. 


\title{
Appendix B. Reprint of invited presentation at MTT-S "High- Performance GaAs/AlGaAs Optical Phase Modulators for Microwave Photonic Integrated Circuits"
}

Hietala, V. M., S. H. Kravitz, M. G. Armendariz, G. A. Vawter, and R. F. Carson, 1994. "High-Performance GaAs/AlGaAs Optical Phase Modulators for Microwave Photonic Integrated Circuits (INVITED)," MTT-S, San Diego, CA, 23-27 May:

\section{High-Performance GaAs/AIGaAs Optical Phase Modulators for Microwave Photonic Integrated circuits}

\author{
Vincent M. Hietala, Stanley H Kravitz, Marcelino G. Armendariz, G. Allen \\ Vawter, \\ and Richard F. Carson \\ Sandia National Laboratories \\ Albuquerque, New Mexico 87185-0603
}

\begin{abstract}
A high-performance high-speed optical phase modulator for photonic integrated circuit (PIC) use is described. Integration of these optical phase modulators into a real system (COMPASS) is also discussed. The optical phase modulators are based on depletion-edge translation and have experimentally provided optical phase shifts in excess of $60 \% / \mathrm{Nm}$ with approximately $4 \mathrm{~dB} / \mathrm{cm}$ loss while simultaneously demonstrating bandwidths in excess of $10 \mathrm{GHz}$.
\end{abstract}

\section{Introduction}

Optical control of microwave systems offers many merits over traditional electrical methods. The primary advantages are improved electromagnetic compatibility of fibers over metal wires, reduced system weight, and lower distribution loss.[1] Additional system-specific benefits are also commonly seen, such as, simplified signal distribution manifold design, efficient high-speed beam forming, and improved feed line flexibility in phased-array antennas.

This paper briefly describes our efforts at Sandia National Laboratories to develop a PIC technology for the control of microwave signals. Our PIC approach is based entirely on "passive" optical components with optical power 
provided by an external laser source. Only passive optical phase shifters are required on-chip to provide useful control functions for microwave applications.

Since high-performance optical phase shifters are critical for this technology this paper will primarily address their required performance and our selected approach. A phased-array antenna control system being pursued at Sandia called COMPASS will be discussed to provide modulator performance guidelines.

\section{COMPASS}

COMPASS [2] (Coherent Optical Monolithic Phased Array Steering System) is an optical concept system which was developed both to address a need for improved phased-array antenna control and to provide a driver for our PIC program. Fig. 1 shows a block diagram of the COMPASS PIC. The PIC is powered by one laser source which is appropriately modulated and split into $\mathrm{N}$ parts and fed through optical fibers to $\mathrm{N}$ antenna elements. The light is then detected to provide a phase controlled RF signal at each antenna element. The RF phase control is performed by optical heterodyning. In any heterodyning process (electrical or optical), phase is preserved between the input signals and the resulting tones. This provides a method of directly mapping an optical phase shift into an RF phase shift.[3] This mapping occurs independent of the RF operating frequency. Therefore, the COMPASS system will allow phase control over a large bandwidth, but instantaneous bandwidth will need to be restricted to exceptable beam squint performance. For narrow band systems (i.e. radar) this will not be an issue.

Fig. 1a shows the Optical Frequency Translator (OFT) portion of the COMPASS PIC. This circuit generates an optical signal which is frequency translated by the RF operating frequency from the input laser. This signal along with a portion of the input light, provides the signals for the heterodyning process. Generating these two optical signals by such a method provides the benefit of phase noise synchronism. Good spectral purity of the RF signals generated at the antennas will therefore be achieved. For a laser coherence length much longer than the path length difference between the two optical signals from the OFT output to the antenna, laser phase noise will be minimized and good RF spectral purity will result. Since the maximum path length error is expected to be less than $10 \mu \mathrm{m}$, a laser with a few $\mathrm{cm}$ coherence length should be adequate.

The OFT operates in an analogous manner to a conventional phaseshift-based SSB modulator.[4] The circuit as shown, can be visualized as two Mach-Zehnder modulators which are operated to produce two double-sideband modulated signals with no carrier. Then by combining the appropriately phased outputs of these two modulators, one of the sidebands can be canceled leaving a lone SSB or frequency translated signal. A more detailed discussion of this circuit along with fabrication was given by lzutsu et al.[5]

The second part of the COMPASS PIC is shown in Fig. 1b. This portion of the circuit divides the optical signals into $N$ parts, introduces the optical phase 
(analog or digital [6]), and provides an interface to $N$ fibers for signal distribution to the $N$ antenna elements. The actual mixing operation occurs in the squarelaw photodetector at the antenna as shown in Fig. 2. The resulting RF signal can then be appropriately signal conditioned and used for the phase-frequency reference for the transmitter or receiver's local oscillator. The two optical signals which produce the RF are sent to the antenna through the same fiber. This reduces fiber length sensitivity to roughly the same requirements as conventional RF waveguide designs. The only phase sensitive paths in the COMPASS system are in the PIC where the two optical channels are routed down different paths. Since these paths are on-chip and by design will be very short and length matched, the system should have excellent temperature stability characteristics for an optical system.

Throughout the COMPASS system optical phase modulators are employed. Phase modulators are the critical component in our PIC technology. These modulators are required to provide high phase-shift-per-unitlength voltage and low optical loss while simultaneously providing large electrical drive bandwidths. For example, in the COMPASS system the OFT section alone requires in excess of $480^{\circ}$ of optical phase shift. Most current optical phase modulator technologies are pushed to provide the meager $180^{\circ}$ phase shift required for Mach-Zehnder amplitude modulators in $\mathrm{cm}$ size chips. Clearly, such approaches would provide little utility in producing a PIC like COMPASS.

Here, through the use of depletion-edge-translation (DET) optical phase modulators we can achieve phase shifts in excess of $60 \% / \mathrm{Nm}$ at $1.3 \mu \mathrm{m}$ optical wavelength. With such an approach, assuming $5 \mathrm{~V}$ operation is desired, the necessary $480^{\circ}$ phase shift can be achieved with simply $1.6 \mathrm{~mm}$ of combined phase modulator length. Naturally, additional device length will be required for the passive optical on-chip interconnects (corners, power splitters/combiners,...), but phase modulator length is expected to still dominate overall chip length.

\section{Depletion-Edge-Translation Modulator}

DET optical phase modulators are based on appropriately combining both electro-optic and carrier effects to provide a maximum phase shift.[7] Fig. 3 shows a diagram of a DET modulator. This modulator lends itself well to integration since passive waveguides and phase modulators have the same exact waveguide structure with the only difference being metal contacts placed on top of the modulators. The light is confined closely to the junction's center to provide a maximum overlap between the electric and optical fields. Note the heavy doping levels used provide a high electric field within the junction. Use of these modulators presents several challenges. First, the heavy doping results in a large terminal capacitance which limits device speed. Secondly, the tight vertical confinement of the light causes critical fabrication issues for proper light guiding and large optical losses in fiber butt coupling. A novel design approach addresses the speed issues, whereas fabrication and packaging issues are addressed elsewhere.[8],[9] 


\section{Distributed Electrode Design}

As discussed above, DET optical phase modulators necessarily suffer from a large terminal capacitance. Capacitance cannot be reduced without lowering the high phase shift of the device which would quickly make COMPASS prohibitively large. Therefore, a distributed approach to the modulator's electrode design was developed. Since a DET modulator has heavy doping, propagation along its electrodes is slow-wave.[10] The first DET modulators fabricated in our laboratory, with no regard for distributed effects, had an estimated RF index $\left(c / v_{p}\right)$ in excess of 20 . Since the optical guides effective index is about 3.5 , velocity mismatch was an issue.

Since an electrical propagation velocity increase is required and the per unit length capacitance is fixed, the only means left to increase the propagation velocity is to reduce the electrode's inductance per unit length $\left(v_{p} \approx 1 / \sqrt{L C}\right)$. This was achieved by changing the device's cross-section to that shown in Fig. 4. The inductance is minimized by reducing the gaps on a slow-wave coplanar waveguide structure[11] to less than $1 \mu \mathrm{m}$. This has experimentally been seen to increase the velocity to levels similar to the light's. The only disadvantage to this approach is the resulting decrease in characteristic impedance caused by the reduction of inductance $\left(Z_{0} \approx \sqrt{L / C}\right)$. Line impedances of about $10 \Omega$ result which will require impedance matching for use in $50 \Omega$ systems.

To date, bandwidths of $10 \mathrm{GHz}$ have been demonstrated with devices similar to that shown in Fig. 4. Though the velocity mismatch has been adequately addressed, electrical propagation losses dominate the modulator response. Simulations performed by quasi-TEM analysis based on finiteelement solutions[12] have indicated that most power dissipation is in the semiconductor regions. In particular, at least half of the line's loss occurred in the p-type caps on the p-mesa to either side of the rib guide (see Fig. 4). These caps are being ion implanted on current designs to minimize this loss. Theoretical bandwidths are then in excess of $30 \mathrm{GHz}$ while still maintaining approximately $60 \% \mathrm{~N} \cdot \mathrm{mm}$ optical phase shift.

\section{Conclusions}

Optical control of microwave systems is possible provided that an adequate PIC technology can be developed. The critical component of such a technology is the optical phase modulator. Optical phase modulators are required to have large phase shifts per unit length.volt in order to allow for realistic sized PICs. Additionally, they must have sufficient bandwidth to operate at the desired microwave system frequency. A depletion-edge-translation modulator under development at Sandia addresses these needs by providing $60 \% \mathrm{~N} \cdot \mathrm{mm}$ optical phase shift with $<4 \mathrm{~dB} / \mathrm{cm}$ optical loss and a $10 \mathrm{GHz}$ bandwidth. Current design considerations predict future bandwidths in excess of $30 \mathrm{GHz}$. 


\section{Acknowledgments}

This work was performed at Sandia National Laboratories supported by the U. S. Department of Energy under contract \#DE-AC04-94AL85000.

\section{References}

[1] E. N. Toughlian, H. Zmuda, and P. Kornreich, "A Deformable Mirror-Based Optical Beam forming System for Phased Array Antennas," IEEE Photon. Technol. Lett., vol. 2, no. 6, pp. 444-446, June 1990.

[2] V. M. Hietala, G. A. Vawter, W. J. Meyer, and S. H Kravitz, "Phased-array antenna control by a monolithic photonic integrated circuit, "SPIE Proc., vol. 1476, pp. 170-175, April 1991.

[3] R. A. Soref, "Voltage-Controlled Optical/RF phase Shifter," J. Lightwave Tech., vol. LT-3, no. 5, pp. 992-998, Oct. 1985.

[4] K. S. Shanmugam, Digital and Analog Communications Systems, pp. 269-272, John Wiley \& Sons, New York, 1979.

[5] M. Izutsu, S. Shikama, and T. Sueta, "Integrated Optical SSB Modulator/Frequency Shifter," IEEE J. Quantum Electron., vol. QE-17, no. 11, Nov. 1981.

[6] G. A. Vawter, V. M. Hietala, S. H Kravitz, "Digital Optical Phase Control in RidgeWaveguide Phase Modulators," IEEE Phot. Tech. Lett., vol. 5, no. 3, March 1993.

[7] J. G. Meddoza-Alverez, L. A. Coldren, A. Alping, R. H. Yan, T. Hausken, K. Lee, and K. Pedrotti "Analysis of Depletion Edge Translation Lightwave Modulators," IEEE J. Lightwave Tech., vol. 6, no. 6, pp. 793-808, June 1988.

[8] G. A. Vawter, J. F. Klem, G. R. Hadley, and S. H Kravitz, "Highly accurate etching of ridge-waveguide directional couplers using in-situ reflectance monitoring and periodic multilayers," Appl. Phys. Lett. 62 (1), pp. 1-3, 4 Jan. 1993.

[9] S. H Kravitz, G. R. Hadley, M. E. Warren, H. R. Wendt, G. A. Vawter, J. C. Word, R. F. Corless, R. F. Carson, M. G. Armendariz, B. E. Hammons, "Waveguide-to-Fiber Coupling Using a Second-Order Grating and an Anamorphic Binary Optic," Proc. IEEE LEOS, pp. 472-473, Nov. 1993.

[10] H. Guckel, P. A. Brennan, and I. Palocz, "A Parallel-Plate Waveguide Approach to Microminiaturized, Planar, Transmission Lines for Integrated Circuits," IEEE Trans. Microwave Theory and Tech., Vol. MTT-15, no. 8, pp. 468-476, August 1967.

[11] H. Hasegawa and H. Okizaki, "M.I.S. and Schottky Slow-Wave Coplanar Striplines on GaAs Substrates," Electron. Lett., vol. 13, no. 22, pp. 663-664, Oct. 1977.

[12] Maxwell 2-D, Ansoft Corporation, Pittsburgh, PA 15219. 

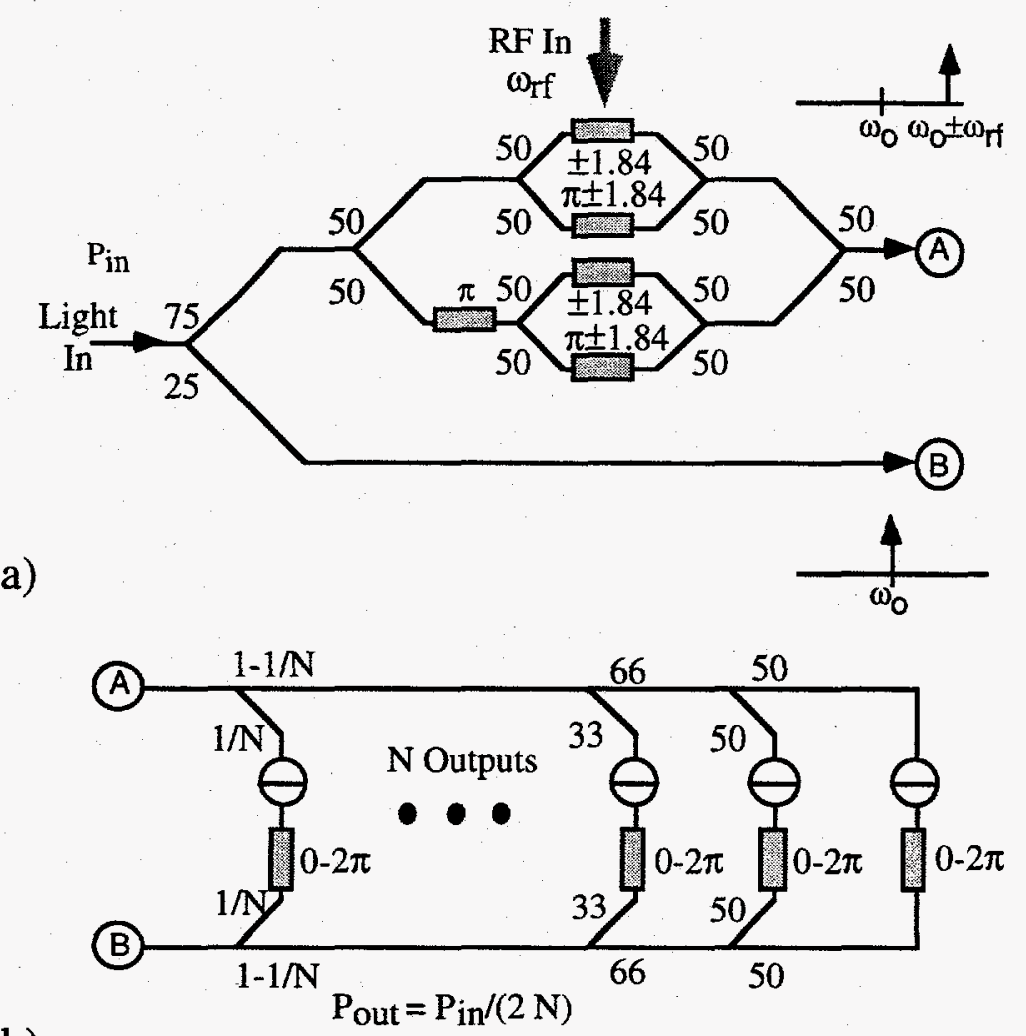

b)

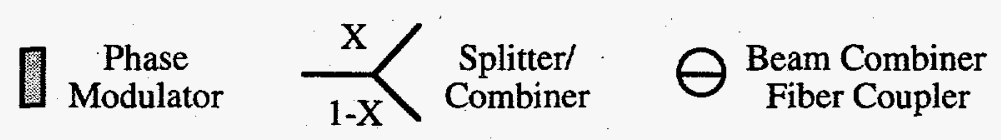

Fig. 1. The COMPASS PIC. a) Optical frequency translator portion. b) Optical power splitters and phase control portion.

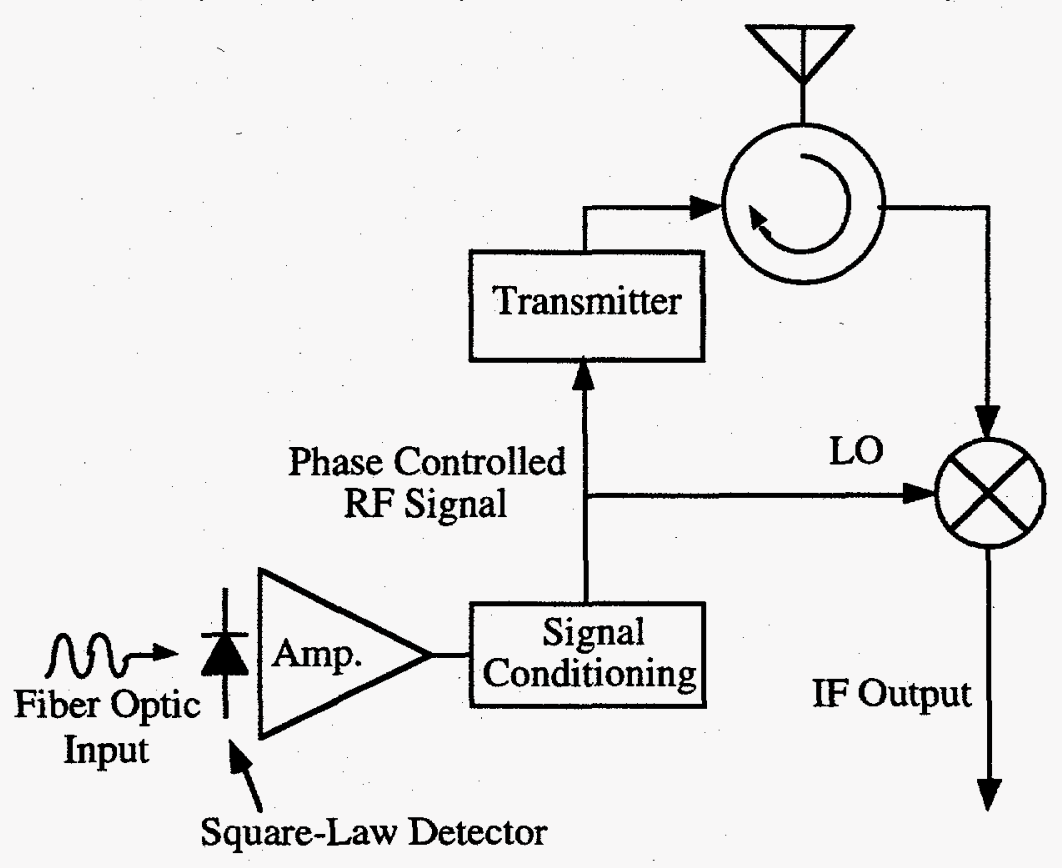

Fig. 2. Transmit/receive module of COMPASS. 


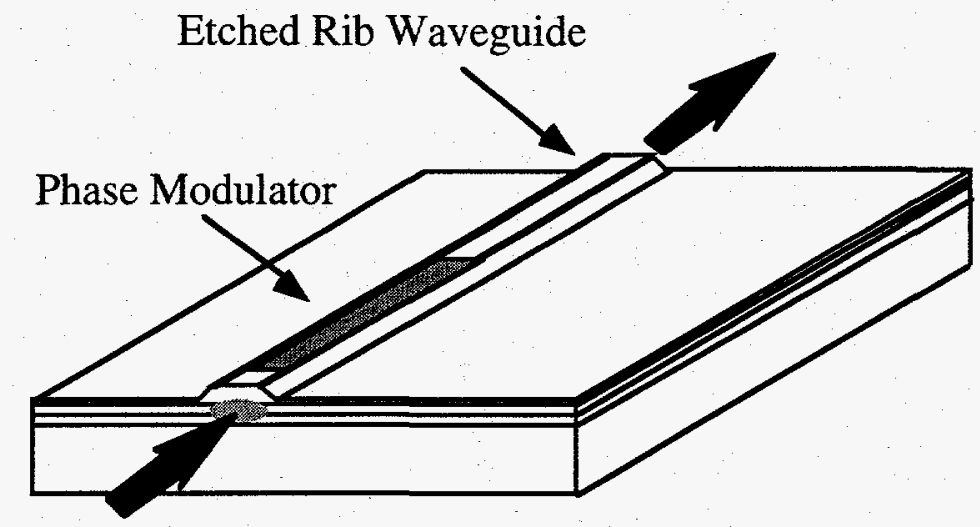

Light

a)

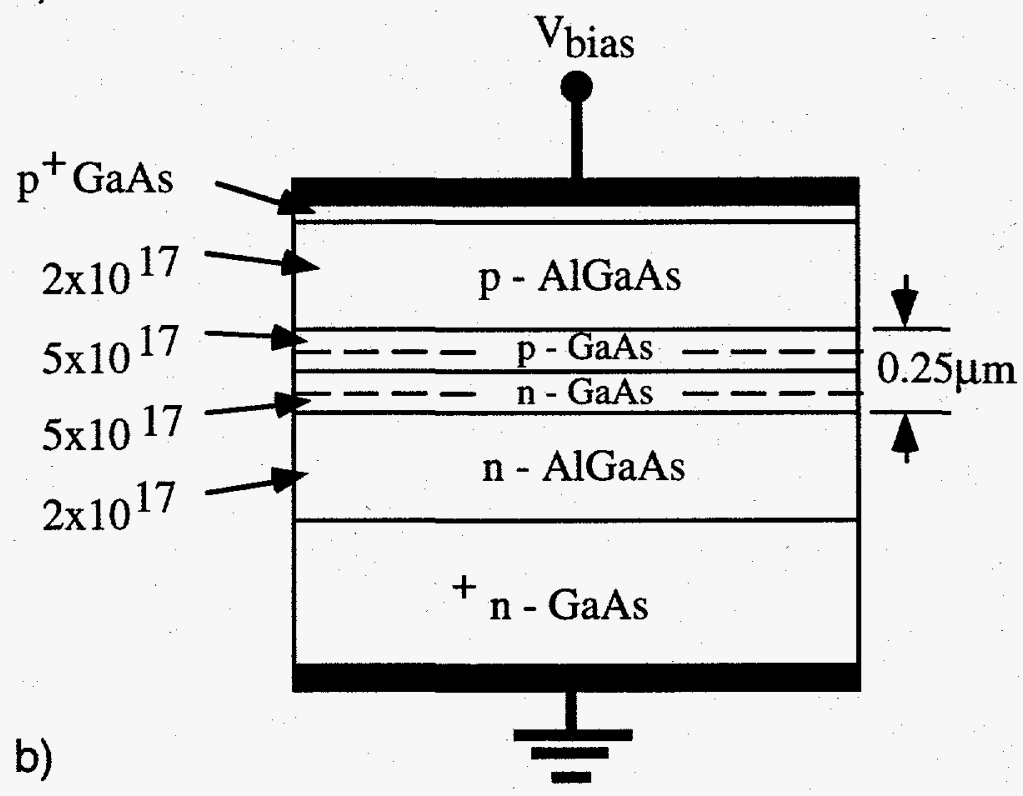

Fig. 3. Depletion-Edge-Translation (DET) optical phase modulator. a) Rib waveguide. b) Modulator cross-section. 


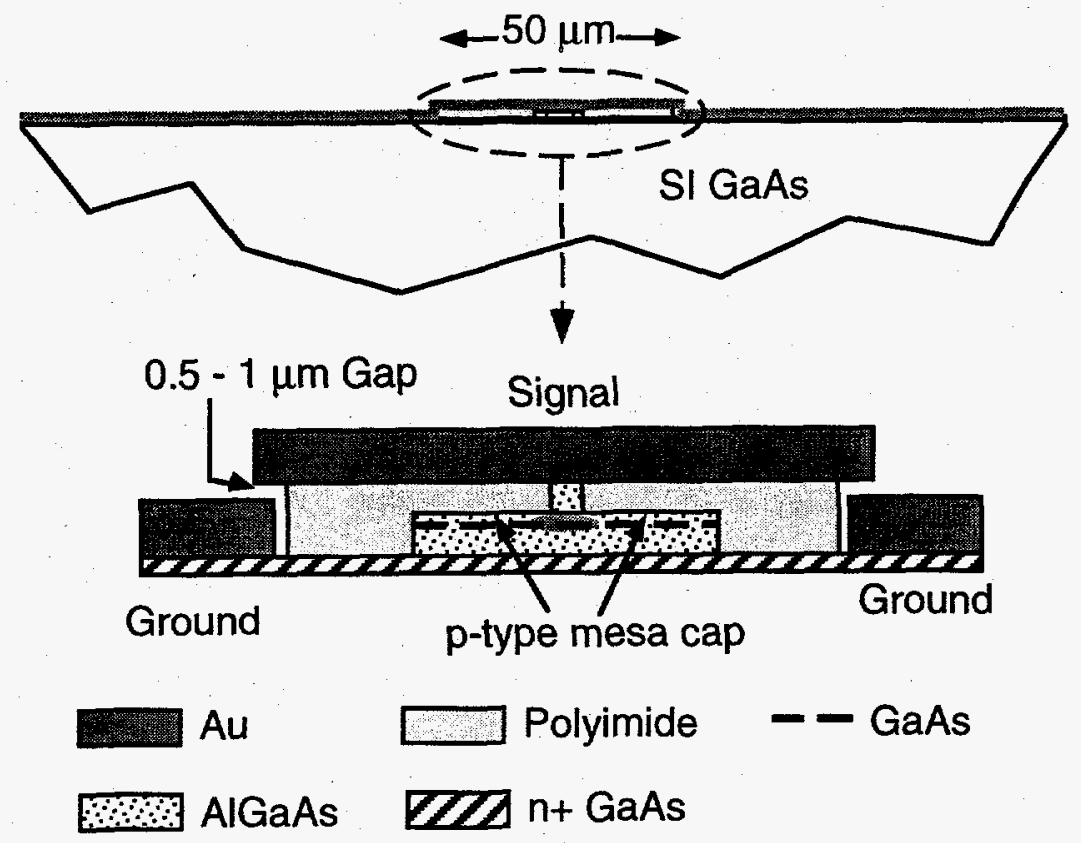

Fig. 4. Cross-section of distributed electrode design of DET modulator. 


\section{Appendix C. LDRD Summary}

\section{Refereed publications resulting for the work: 4}

Hietala, V. M., S. H. Kravitz, M. G. Armendariz, G. A. Vawter, and R. F. Carson, 1994. "High-Performance GaAs/AlGaAs Optical Phase Modulators for Microwave Photonic Integrated Circuits (INVITED)," MTT-S, San Diego, CA, 23-27 May.

Vawter, G. A., J. F. Klem, and R. E. Leibenguth, 1994. "Improved Epitaxial Layer Design for Real-Time Monitoring of Dry-Etching in III-V Compound Heterostructures With Depth Accuracy of $\pm 8 \mathrm{~nm}, " \mathrm{~J}$. of Vacuum Science and Technol. A, Jul/Aug.

Vawter, G. A., J. F. Klem, G. R. Hadley, and S. H. Kravitz, 1993. "Highly Accurate Etch Depth Control of Ridge-Waveguide Directional Couplers Using InSitu Reflectance Monitoring and Periodic Multi-Layers," Appl. Phys. Lett., 62(1), pp.1-3, 4 January.

Armendariz, M. G., V. M. Hietala, and D. R. Myers, 1994. "High-Speed Optoelectronics at Sandia National Laboratories," Microwave Journal, June.

\section{All other reports and publications resulting from the work: 6}

Hietala, V. M., S. H. Kravitz, G. A. Vawter, M. G. Armendariz, and R. F. Carson, 1993. "High-Speed High-Performance Optical Phase Modulators," Paper presented at the Progress in Electromagnetic Research Symposium, Pasadena, 12-16 July.

Hietala, V. M., S. H. Kravitz, M. G. Armendariz, G. A. Vawter, R. F. Carson, and R. E. Leibenguth, 1994. "High-performance GaAs/AIGaAs optical phase modulators for microwave/photonic integrated circuits," presented at Optoelectronic Signal Processing for Phased Array Antennas IV, SPIE OE LASE '94, Los Angeles, CA, 22-28 January.

Vawter, G. A., and R. Hadley, 1994. "III-V Compound Semiconductor StripLoaded Waveguide Devices for PICs: Design for Minimum Crosstalk and High Density (INVITED)," SPIE International Symposium on Optoelectronic and Microwave Engineering, Los Angeles, CA., 22-28 January.

Vawter, G. A., G. R. Hadley, J. R. Wendt, and J. F. Klem, 1994. "An Integrated Optical X-Y Coupler for Phase-Sensitive Optical Power Combining and Suppression of Radiated Light," CLEO '94, Anaheim, CA, 8-13 May. 
Vawter, G. A., 1993. "Epitaxial Layer Design for in-situ Monitoring of Dry-Etching in III-V Compound Heterostructures," Paper presented at AVS 40th National Symposium and Topical Conferences, Orlando, 15-19 November.

Kravitz, S. H., G. A. Vawter, V. M. Hietala, M. B. Snipes Jr., M. G. Armendariz, R. F. Carson, B. E. Hammons, and R. E. Leibenguth, 1994. "Fabrication of High-Performance GaAs/AIGaAs Optical Phase Modulators for Microwave/Photonic Integrated Circuits," presented at Optoelectronic Signal Processing for Phased Array Antennas IV, SPIE OE LASE '94, Los Angeles, CA, 22-28 January.

Number of patent disclosures: 0

Number of patent applications: 0

Number of patents: 0

Number of Copyrights: None

Number of students: None

Number of post docs: None

Number of permanent technical or scientific staff: None

Number of awards (and their names): None

Number of new non-LDRD funded projects and their amounts: None 
Distribution:

1 MS0320 LDRD Office, 1010

1. MS1079 P. S. Peercy, 1300

$\begin{array}{lll}1 \text { MS1078 } & \text { D. R. Myers, } 1303\end{array}$

1 MS1425 S. J. Martin, 1315

1 MS1078 $\quad$ H. T. Weaver, 1321

1 MS0603 T. E. Zipperian, 1322

1 MS0603 J. Y. Tsao, 1311

1 MS0603 A. Owyoung, 1312

2 MS0603 T. A. Plut, 1322

$\begin{array}{lll}2 . M S 0603 & \text { G. A. Vawter, } 1322\end{array}$

15 MS0603 V. M. Hietala, 1322

2 MS0603 J. R. Wendt, 1322

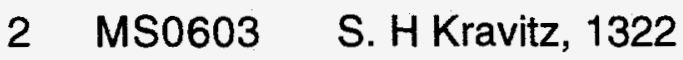

$2 \begin{array}{ll}\text { MS0874 } & \text { M. G. Armendariz, } 1342\end{array}$

1 MS0874 $\quad$ W. J. Meyer, 1342

1 MS9018 Central Technical Files, 8523-2

5 MS0899 Technical Library, 13414

1 MS0619 Print Media, 12615

2 MS0100 Document Processing, 7613-2

For DOE/OSTI 